\title{
A generalised power-law formulation for the modelling of damping and stiffness nonlinearities
}

\author{
Marco Civera a,*, Stefano Grivet-Talocia b, Cecilia Surace c, and Luca Zanotti Fragonara d \\ a Department of Mechanical and Aerospace Engineering, Politecnico di Torino, 10129 Turin, Italy \\ b Department of Electronics and Telecommunications, Politecnico di Torino, 10129 Turin, Italy \\ c Department of Structural, Building and Geotechnical Engineering, Politecnico di Torino, 10129 Turin, Italy \\ d Centre for Autonomous and Cyber-Physical Systems, Cranfield University, Cranfield, \\ Bedford MK43 0AL, UK
}

\begin{abstract}
In this paper, a single-degree-of-freedom dynamic model is described, with displacement- and velocitydependent nonlinearities represented by power laws. The model is intended to support the dynamic identification of structural components subjected to harmonic excitation. In comparison to other analytical expressions, the data-driven estimation of the nonlinear exponents provides a large versatility, making the generalised model adaptable for a wide number of different nonlinearities in both stiffness and damping. For instance, the proposed damping formulation can naturally accommodate air drag (quadratic) damping as well as dry friction. Differently to purely data-driven methods (e.g. black boxes), the obtained model is fully inspectable. The proposed formulation is here applied to the large oscillations of a prototype highly flexible wing and fitted on its steady state response in the frequency domain. These large-amplitude flap-wise bending oscillations are known to be affected by nonlinearities in both the stiffness (nonlinear hardening) and the velocity-dependent damping terms. The model is validated against experiments for different structural configurations and input amplitudes, as both these nonlinearities are energy-dependent.
\end{abstract}

Keywords: Nonlinear Dynamics, Nonlinear Hardening, Nonlinear Damping, Large Vibrations, Nonlinear System identification, High-Aspect-Ratio wing.

* Correspondence to: Marco Civera, Department of Mechanical and Aerospace Engineering, Politecnico di Torino, Corso Duca degli Abruzzi 24, 10129 Turin, Italy

E-mail address: marco.civera@polito.it 


\section{Symbols}

$a$ Output displacement amplitude at steady state.

$c, c_{2}, c_{\eta+1}$ Linear damping coefficient, quadratic damping coefficient, and generalised nonlinear damping coefficient.

$F$ Input force amplitude.

$f_{k}(x)$ Nonlinear stiffness term

$f_{d}(x)$ Nonlinear damping term

$k, k_{3}, k_{\rho+1}$ Linear stiffness coefficient, cubic stiffness coefficient (Duffing term), and generalised nonlinear stiffness coefficient.

$m$ Mass.

$x, \dot{x}, \ddot{x}$ displacement, velocity, and acceleration of the forced system.

$\alpha, \alpha_{e q}, \alpha_{e f f}$ Mass-normalised linear stiffness, equivalent mass-normalised linear stiffness, and effective massnormalised linear stiffness.

$\beta$ Mass-normalised cubic stiffness coefficient.

$\gamma$ Mass-normalised input force amplitude (note: equal to the input acceleration amplitude).

$\delta, \delta_{e q}, \delta_{e f f}$ Mass-normalised linear damping, equivalent mass-normalised linear damping, and effective massnormalised linear damping.

$\zeta, \zeta_{\text {eq }}, \zeta_{\text {eff }}$ Linear damping ratio, equivalent linear damping ratio, and effective linear damping ratio.

$\eta$ Exponent of $|\dot{x}|^{\eta}$ in the nonlinear damping term $f_{d}(x)$.

$\theta$ Vector of the (linear and nonlinear) parameters of the system.

$\kappa$ Mass-normalised quadratic damping coefficient.

$\rho$ Exponent of $|x|^{\rho}$ in the nonlinear stiffness term $f_{k}(x)$.

$\omega$ and $\omega_{n}$ Angular frequency and natural angular frequency

$\Omega$ Angular frequency of the driving force.

\section{Introduction}

Nonlinearities are ubiquitous in natural and man-made structures. For instance, they are almost always encountered in the Ground Vibration Tests (GVTs) of aircraft (see e.g. [1],[2]). These are mainly due to clearance nonlinearities or caused by mechanical components as e.g. the mounting brackets. Noise-like distortions in the frequency response caused by material, geometrical, or other sources of nonlinearity are the norm for civil buildings and other mechanical systems (see e.g. [3]) as well. It is also widely known that damaged structures tend to behave nonlinearly due to the phenomenon of the breathing crack mechanism [4]. An exhaustive discussion on the whole argument of Nonlinear System Identification (NLSI) can be found in the very comprehensive reviews of Kerschen et al [5] and Noël 
et al [6] and in the book of Worden and Tomlinson [7].

Under certain circumstances, it is possible to neglect, by-pass, or approximate the effects of nonlinearities, avoiding time- and resource-consuming investigations. For instance, linearisation is currently a common practice for structural engineers, if one accepts the inevitable errors of applying a linear model to a nonlinear system. In this sense, it is possible to define the best linear approximation of a nonlinear system and to define the limits of validity of such a simplified model (e.g. [8,9]).

However, due to unmodeled nonlinearities, the difference between the estimated and the encountered natural frequencies in regular aircraft can raise from a $3 \%$ deviation up to an 8-9\% error [10]. Being the GVT tests carried out on the grounded plane and in still air, the difference between the expected and the actual dynamic behaviour can only increase in-flight where flow speed-dependent aeroelastic loads are added. This not only compromises the intended response of the structure but negatively affects the assessment of flutter speed limits and may cause unexpected aerodynamic interactions and/or modal coupling, further resulting in stability issues.

On the other hand, some mechanical systems are purposely designed to behave nonlinearly. Two classic examples are automotive shock absorbers [11,12] and nonlinear energy harvesters [13]. In the latter case, the nonlinearity allows producing piezoelectric energy more efficiently than in the linear case. Indeed, while often only regarded as an issue or an unwanted complication, nonlinear dynamics may be profitable if properly exploited.

It must be said that nonlinearities are often associated with large model uncertainties [6]; this ideally will require case-by-case, ad hoc experimental campaigns. An example of Aerospace application is, e.g., the testing of all joints of the structure [14]. Whenever this is not economically sustainable, simplified analytical expressions can be used for some specific nonlinear models. For instance, the nonlinear stiffness is generally modelled as a function of the cubic term of displacement, known as the Duffing term [15]; this and similar models have been widely investigated (e.g. [16,17]). Nonlinear damping is somehow relatively less studied, even if it can become predominant respect to the stiffnessrelated source of nonlinearity, especially due to increased air drag on fast-moving structures. For the specific case of large vibrations, the nonlinear damping is generally approximated as a quadratic 
function of the velocity [18]. Some other models have been proposed in the literature as well. For instance, Cao and Ge [19] proposed a dynamic equation with nonlinear air damping and added mass for a vertically vibrating bridge deck section. They defined amplitude-dependent nonlinear terms for mass and viscous damping, but no explicit parameters for the nonlinear stiffness. They argued that, since the effects of additional mass and additional stiffness cannot be distinguished from frequency alone, the nonlinear air-added mass accounted for both effects. Yet this is known to be valid only for linear dynamics and the coupling between mass and stiffness in nonlinear conditions are still a matter of research (especially for the Authors of this study).

Noteworthy, most of the research in the area of nonlinear damping is focused on microcantilever beams for energy harvesting or other micro- or nano-mechanical aims [20,21]; yet these may have different behaviours as they have much smaller surface subject to air drag. For that specific context, a useful review of formulations for air drag-induced additional damping can be found in Reference [22]. In any case, all analytical formulations should incorporate as much as possible any available form of prior knowledge on the system, including its known first-principle physics. Dedicated tuning parameters, which are context-dependent by definition, are then left free to vary and can be calibrated for the given validation domain of interest.

In this context, a generalised analytical formulation, capable to adapt for different applications rather than being limited to a single specific intent, will be very helpful. Here, the physical principles of the linear equation of motion are retained and then complemented by additional nonlinear terms. These latter ones are derived from the commonly accepted formulations described above, i.e. the cubic nonlinear stiffness and the quadratic the nonlinear damping, which have been here generalised for any arbitrary integer or non-integer exponent. This is intended to improve their capacity to cope with different physical phenomena, differently from any other methodology found in the literature. It is also demonstrated how air drag-induced (quadratic) damping and dry friction can be both described by the proposed generalised term by adapting its parameters. The formulation departs from a Harmonic Balance (HB) approach, not too dissimilar to the one proposed in similar works, e.g. by Amabili [23], truncated to the first term for reasons that will be better explained in the dedicated 
paragraphs. This is well-suited for this study, as HB is considered the analytical analogue of the stepped-sine test procedure ([6]) experimentally performed on the case study of interest.

The rest of this paper is structured as follow. Section 2 introduces the specific case study investigated. In Section 3, the classic models for the nonlinear stiffening and damping are presented; the proposed expressions are then derived from them. Some useful remarks are reported as well. Section 4 discusses the fitting procedure over the experimental data and reports the result for the proposed models, also in comparison with the state-of-the-art model (Duffing stiffness and quadratic damping). Key findings and results are outlined and illustrated. Thepaper ends with theConclusions; somelimitations and caveats are reported as well.

\section{Case study: the highly flexible HAR wing prototype}

The specific case study analysed here is the highly flexible, High-Aspect-Ratio (HAR) XB-1 prototype wing developed in the framework of the Cranfield University Beam Reduction and Dynamic Scaling (BeaRDS) Programme [24]. The goal is to produce a nonlinear Reduced Order Model with a Single Degree of Freedom (SDoF) able to capture the dynamic response of the slender wing. The use of an ideally-zero-stiffness skin [24] was intended to transfer all static and aerodynamic loads to the spar; thus, the spar alone was experimentally tested.

Results from experiments performed on the same structure were already deeply investigated by the Authors [25-28]; from those earlier investigations, both nonlinear hardening and nonlinear damping were found to be non-negligible at large oscillations. The difficulties found in modelling these nonlinearities were the driving cause of this research. Indeed, the current trend for wing design leads to lighter and more flexible elements, subject to larger oscillations; understanding and modelling the inherent nonlinear dynamics will be useful to de-risk dangerous aeroelastic effects [29], as well as to potentially exploit the increased flexibility to suppress flutter, alleviate aerodynamic loads, and increase roll manoeuvrability at high speed [30,31].

It is fundamental to remember that for any nonlinear system, the frequency response of the steady state regime can only be experimentally reached through quasi-stationary procedures such as 
the step sine method [32]. This is very well-known and can be appreciated for instance by comparing the Frequency Response Function of a stepped or very slowly swept sine and of a random noise input around any resonant frequency of a nonlinear system. Only the stepsine can maintain and follow a stable, steady state regime at any frequency. This may be achieved also by means of a very slow chirp rate if the system is not strongly nonlinear. The random noise will only provide a linearised approximation of the same, with noise-like distortions as a by-product of the nonlinearity. Therefore, for the rest of this discussion, only the steady state response of the HAR wing will be considered.

The specifics of the HAR wing spar (Figure 1.a) are reported in Table 1. The experimental data were recorded at the facilities of Cranfield University and come from 5 levels of input amplitude and two structural configurations, with and without sensors attached. None of the structure's modes was found to be involved in an internal resonance with the other ones, and they are all well-distanced. Finally, only the first mode was found to produce displacements large enough to cause nonlinearities in the response; this is described in detail in Reference [26].

[Table 1 about here.]

[Figure 1 about here.]

\subsection{The experimental setup}

The experimental setup is pictured in Figure 1.b. Recordings were performed with a Polytec $®$ OFV-505 single point Laser Doppler Vibrometer (LDV). The input was applied as an external acceleration at the clamped end through a Data Physics® Signal Force modal shaker and its DP760 close-loop control software. The software was set to increase the input frequency of $1 \mathrm{mHz}$ at any step and to dwell until steady state was reached before sweeping to the next one. The duration of the frequency steps was not constant; an automatic settling time option was used. With this setting, the software takes output amplitude measurements at any cycle of the input until the difference in the value of two consecutive measurement does not exceed a certain selected tolerance (here, 1\%) [33]. Unloaded and sensor-loaded configurations were studied, to address specifically the effects of the 
sensors' added masses on the nonlinear response of the system. In the latter case, four Raspberry $\mathrm{PI}($ inertial measurement units (IMUs) were applied. The sensor locations can be seen in Figure 1.c. Please note that only the LDV data were used as output channel, as the experiment lasted too long to be recorded via the deployed sensor network, which had a limited capacity. Thus, the sensors must be considered only as added weights.

The data were recorded for five levels of increasing input amplitude (reported in Table 2), single input single output, with a stepped sine approach (for 200 frequency steps). Two typologies of data were directly extracted from any experiment: the acceleration time histories and the amplitudes of the steady state Frequency Response Functions (FRFs), defined between the input acceleration (a pure sinusoid with angular frequency $\Omega$ ) and the steady state output displacements (which are periodic but not purely sinusoidal due to higher harmonics). These latter data are here indicated by $|H(s=j \Omega)|$ where $j$ is the imaginary unit; they were automatically preprocessed by the machine and returned to the user. All measurements are intended as Peak-to-Peak (P-P).

It can be seen from Figure 2 how the resonance (marked by red dots and called at phase quadrature between the input and the output) moved downward and to the higher frequencies for increasing levels of input amplitude, i.e. for increasing system energy. These are evidence, respectively, of amplitude-dependent nonlinear damping and hardening. For the highest amplitude level (2.46 $\left.\mathrm{m} / \mathrm{s}^{2} \mathrm{P}-\mathrm{P}\right)$ the LDV started to suffer from clipping in the unloaded case, resulting in numerous spikes. The issue was only partially solved by removing the spikes manually.

[Table 2 about here.]

[Figure 2 about here.]

A major practical concern derives from the size of the experimental data. Indeed, the step sine test is notoriously long, as per any step it requires to dwell the system until it reaches the steady state before sweeping to the next step. For very low frequencies as the one inspected here (between $5 \cdot 3$ and $5.5 \mathrm{~Hz}$ ), waiting for the steady state to be reached can take several seconds, or even some 
minutes, per step. That leads easily to more than one hour of recording per experiment. For this reason, the preprocessed amplitude response functions (indicated by the dark blue lines in Figure 2) were preferred as they carried the same information in a much more compact way.

\subsection{The main sources of nonlinearities}

As mentioned, two main sources of nonlinearities have been detected from the acquired data: nonlinearity in the stiffness term (displacement-dependent) and nonlinearity in the viscous damping term (velocity-dependent). Specifically, nonlinear hardening was noticed from the leaning of the backbone curve to the higher frequencies [26]. From the same previous studies, clear signs of nonlinear damping were noticed in the free decay from dwelling at the first mode (Figure 3.a), visible both in the nonlinear log-Hilbert transform (Figure 3.b) and in the ovalisation of the Nyquist plot (Figure 3.c and Figure 3.d). The fact that the nonlinear damping increased for increasing input energy levels, rather than the opposite, pointed undoubtedly to an additional nonlinear viscous term, typical of fast-moving objects in fluids, as dry friction-induced nonlinearity (which is particularly prevalent in demountable structures [7]) conversely increases when the input amplitude decreases.

[Figure 3 about here.]

The harmonic distortion of the recorded output was found to be very limited. This is quite understandable as the system shows only weak nonlinearities. This can be appreciated by performing a short-time Fourier transform around the natural frequency at any input energy level. It was found that the first superharmonic was two orders of magnitude lower than the corresponding natural frequency. It is thus possible to consider the equivalent linearised response as a single equivalent sinusoid, which means that the system can be modelled with sufficient precision by performing a Harmonic Balance approximation truncated to the first term.

\subsection{Linear system identification}

Sweep (not stepped) sine tests were performed from $4.500 \mathrm{~Hz}$ to $6.000 \mathrm{~Hz}$ with a chirp rate of +0.005 $\mathrm{Hz} / \mathrm{s}$ and input P-P amplitude of $0.5 \mathrm{~m} / \mathrm{s}^{2}$ (low enough to ensure the linearity of the response) on both the unloaded and the sensor-loaded configuration, considering the whole spar as an equivalent linear SDoF 
oscillator. The resulting values of mass, linear stiffness, and linear damping are reported in Table 3. The linear System Identification (SI) was performed employing a Fast Relaxed Vector Fitting (FRVF) algorithm [34-36], atechnique recently adapted for mechanical systems [37]. For the loaded configuration, the linear SI confirmed the expected increase in mass but also detected an increase in the linear stiffness, quite surely due to slight differences in rearranging the experimental setup after the first set of experiments. The increases in both mass and stiffness partially compensated one another on the vibrational response of the spar. A slight difference in damping was found as well.

[Table 3 about here]

\section{The nonlinear analytical models}

In this Section, the nonlinear models considered are presented. Specifically, it is necessary to briefly recall the two most classic models for the large-amplitude dynamics of the beam- and plate-like transverse flap-wise oscillations, to provide the needed background. These are the already mentioned cubic stiffness and quadratic damping models. The rationale is to have them accounting for, respectively, the hardening behaviour and the increased viscous damping induced by the air drag. These two components are then combined in one mixed model. This is valid since the nonlinearities in stiffness and damping are known to not interfere with each other and are therefore additive.

\subsection{The SDoF oscillator with cubic stiffness (Duffing model)}

The Duffing SDoF oscillator is a very well-known system (see e.g. Nayfeh and Mook [32], Chapters 1.4.1 and 4.1), which differs from the classic linear equation of motion only by the additional nonlinear term $f_{k}(x)=k_{3} x^{3}$. Therefore, only its basic definition is here reported for the sake of its generalisation, which will be performed in the next Sections. In the (linearly damped) SDoF case, its equation of motion, when divided by the mass of the system $m$, becomes

$$
\ddot{x}+\delta \dot{x}+\alpha x+\beta x^{3}=\gamma \cos (\Omega t)
$$

where $\delta=\frac{c}{m}$ and $\alpha=\frac{k}{m}$ are, respectively, the mass-normalised linear terms of viscous 
damping and stiffness, $\beta=\frac{k_{3}}{m}$ is the mass-normalised Duffing term, and $\gamma=\frac{F}{m}$ is the input acceleration (being $F$ the external driving force, which is harmonic with a pulsation of $\Omega$ ). The variable of the equation is the displacement $x$, along with its first and second time derivatives $\dot{x}, \ddot{x}$. The steady state response of the Duffing oscillator can be balanced by a sum of harmonic functions, i.e. approximated via Harmonic Balance, in this case truncated to the first order. The rationale is that a solution of the Duffing equation exists in the form of $x=q \cos (\Omega t)+p \sin (\Omega t)=a \sin (\Omega t-\varphi)$, where $q^{2}+p^{2}=a^{2}$ is the square of the amplitude of the resulting harmonic response and $\tan (\varphi)=\frac{p}{q}$ its phase. By substituting this tentative solution into Equation 1 and neglecting the superharmonics at $3 \Omega$, an equivalent harmonic can be found (the exact formulation can be retrieved in Nayfeh and Mook [32], Chapter 4.1). What is essential to recall here is the definition of the equivalent term of stiffness $k_{e q}$, which can be grouped together with its linear counterpart such that

$$
\alpha_{e f f}=\frac{k}{m}+\frac{k_{e q}}{m}=\alpha+\frac{3}{4} \beta a^{2}
$$

Equation 2

is the resulting mass-normalised effective stiffness. A discussion about the stability of the response would be needed at this point since more states can exist at different energy levels for the same frequency under the proper conditions. However, this aspect does not affect the weak nonlinearities investigated here and is therefore omitted, due to space limitations.

\subsection{The SDoF oscillator with quadratic damping}

It is known that a body moving through a viscous fluid is subject to a drag force proportional to the speed of its motion. This is also the case for a structure oscillating in still air. At low and very low Reynold numbers (Re), the relationship between the body velocity and the drag force follows the Stokes' law and is therefore linear. In that specific case, without any further source of damping, the coefficient $c$ is supposed to model the particular body geometry and the properties of the fluid (e.g. the air) in which it is immersed. In reality, this coefficient accommodates many other unmodelled contributions to the structural damping, which for mathematical convenience are generally assumed 
as linearly proportional to the mass and stiffness of the system (e.g. the well-known Rayleigh damping model). This is the classic model of (linear) viscous damping conventionally applied in structural dynamics.

At high Re, the drag forces are nearly proportional to the square of velocity. Therefore, the quadratic damping is the default choice. In this case, the single source of nonlinearity is incorporated in $f_{d}(\dot{x})=c_{2} \dot{x}|\dot{x}|$, where $\dot{x}|\dot{x}|$ is needed to account for the sign of $\dot{x}$, to ensure that the force is always opposed to the velocity. Mass-normalising the quadratic damping term as $\kappa=\frac{c_{2}}{m}$, the equivalent and the effective (mass-normalised) linear damping can be then defined as ([7], Chapter $3.6)$,

$$
\delta_{e f f}=\frac{c}{m}+\frac{c_{e q}}{m}=\delta+\frac{8}{3 \pi} \kappa \Omega a
$$

This concludes the background needed for the rest of this paper. The definition of the modulation equations, eigenvalues, and related concepts for both the Duffing and the quadratic damping model can be found in any relevant textbook (as the previously mentioned [32]).

\subsection{The SDoF oscillator with cubic stiffness and quadratic damping}

It is possible, at this point, to add together the two sources of nonlinearities. The mixed cubic stiffness - quadratic damping formulation can be considered as the default model for the nonlinear large transverse oscillations of thin structures and is very similar, to a certain extent, to the approach followed e.g. in Reference [38] or by other analytical models with combined stiffness and damping nonlinearities, such as the ones reported in Reference [39] or [40] for the transverse and the axial vibrations of an SDoF system (in this order).

However, this classic model has been proven unfitted to describe the nonlinear response of the wing spar here investigated. This will be better detailed in the following Sections. To overcome this issue, the generalisation of the nonlinear models to any arbitrary power is proposed. Therefore, the aim is to provide the most general possible definition of the effective stiffness and damping $\alpha_{\text {eff }}$ 
and $\delta_{e f f}$.

\subsection{The SDoF oscillator with generalised nonlinear stiffness}

The $f_{k}(x)$ term, previously considered as a cubic function, can assume a generalised (yet still displacement dependent) form as

$$
f_{k}(x)=k_{\rho+1} x|x|^{\rho}
$$

Equation 4

where Equation 4 defines the general form of a signed (odd) power function with an arbitrary (not necessarily integer nor positive) exponent $\rho$-Only odd functions $f_{k}(x)$ should be considered, discarding even functions for the stiffness, since these latter ones do not have a physical sense in this context. By applying the first-order HB procedure, the generalised equivalent stiffness becomes

$$
k_{e q}=\frac{1}{a \pi} \int_{0}^{2 \pi} f_{k}(a \cos (\theta)) \cos (\theta) d \theta=\frac{1}{a \pi} \int_{0}^{2 \pi} k_{\rho+1}|a \cos (\theta)|^{\rho} a \cos ^{2}(\theta) d \theta
$$

this can be further simplified as

$$
k_{e q}=\frac{k_{\rho+1}}{\pi} \int_{0}^{2 \pi}|a \cos (\theta)|^{\rho} \cos ^{2}(\theta) d \theta=4 \frac{|a|^{\rho} k_{\rho+1}}{\pi} \int_{0}^{\pi / 2} \cos (\theta)^{\rho+2} d \theta
$$

Equation 6

It is possible to integrate by substitution, since

$$
\begin{aligned}
& \xi=\cos (\theta), d \xi=-\sin (\theta) d \theta \rightarrow \\
& \theta=\cos ^{-1}(\xi), d \theta=-\frac{1}{\sqrt{1-\xi^{2}}} d \xi
\end{aligned}
$$

where $\cos ^{-1}(\xi)$ indicates the arccosine of $\xi$. Then one obtains

$$
k_{e q}=4 \frac{|a|^{\rho} k_{\rho+1}}{\pi} \int_{1}^{0} \xi^{\rho+2} \cdot\left(-\frac{1}{\sqrt{1-\xi^{2}}}\right) d \xi=4 \frac{|a|^{\rho} k_{\rho+1}}{\pi} \int_{0}^{1} \frac{\xi^{\rho+2}}{\sqrt{1-\xi^{2}}} d \xi
$$

defined $\forall \rho>-3$. The solution of the integral is 


$$
k_{e q}=\left(\frac{\Gamma\left(\frac{3+\rho}{2}\right)}{\Gamma\left(2+\frac{\rho}{2}\right)}\right) \frac{2}{\sqrt{\pi}} k_{\rho+1}|a|^{\rho} \quad \forall \rho>-3
$$

Where $\Gamma(\square)$ is the Gamma function, which extends the factorial $\Gamma(n)=(n-1)$ ! to non-integer values of $n$. To proof that the cubic stiffness (i.e. the Duffing term) is a specific case of this more general definition, it is just needed to substitute $\rho=2$; one obtains

$$
k_{e q}=\left(\frac{\Gamma\left(\frac{5}{2}\right)}{\Gamma(3)}\right) \frac{2}{\sqrt{\pi}} k_{3} a^{2}=\left(\frac{3 \sqrt{\pi}}{4} \cdot \frac{1}{2}\right) \frac{2}{\sqrt{\pi}} k_{3} a^{2}=\frac{3}{4} k_{3} a^{2}
$$

Equation 10

Dimensionally, since $k_{e q}$ and $a^{\rho}$ are, respectively, $\left[\frac{N}{m}\right]$ and $\left[m^{\rho}\right], k_{\rho+1}$ is defined in terms of $\left[\frac{N}{m^{\rho+1}}\right]$ (or, equivalently, of $\left[\frac{k g}{m^{\rho} s^{2}}\right]$ ).

It is possible to write the generic steady state solution of the investigated SDoF system as

$$
\frac{\Omega}{\omega_{n}}=1+\frac{1}{2 m \omega_{n}^{2}} k_{e q} \pm \sqrt{\frac{\gamma^{2}}{\left(2 \omega_{n}^{2}\right)^{2} a^{2}}-\zeta^{2}}
$$

Equation 11

which is valid for any definition of $k_{e q}$ and not only limited to the one of interest here defined in Equation 9. Note that in Equation 11 the linear damping is expressed in the form of its equivalent non-dimensional damping ratio $\zeta=c / c_{c}$, with critical damping $c_{c}=2 m \omega_{n}$, for convenience.

One can further investigate the numerical values assumed by the equivalent coefficient by varying $\rho$; this can be conveniently done by defining a function $g(\rho)$ such that 


$$
g(\rho)=\frac{k_{e q}}{k_{\rho+1}|a|^{\rho}}=\left(\frac{\Gamma\left(\frac{3+\rho}{2}\right)}{\Gamma\left(2+\frac{\rho}{2}\right)}\right) \frac{2}{\sqrt{\pi}} \quad \forall \rho>-3
$$

One can see that $\lim _{\rho \rightarrow-3} g(\rho)=+\infty$ and $\lim _{\rho \rightarrow+\infty} g(\rho)=0$. Moreover, when $\rho=0$, the function becomes $g(\rho)=1$, that is to say, it is reduced to its linear case, with $k_{e q}=k_{1} \cdot|x|^{0}=k_{1}$ and as expected $k_{1}=k$. Apart from the constant term $\frac{2}{\sqrt{\pi}}, g(\rho)$ is defined by the ratio of two Gamma functions. For non-negative values of $\rho$, a quotient of Gamma functions can be approximated by the function

$$
\left(\frac{\Gamma\left(\frac{3+\rho}{2}\right)}{\Gamma\left(2+\frac{\rho}{2}\right)}\right) \cong\left(\frac{\rho}{2}\right)^{-\frac{1}{2}}
$$

Equation 13

with increasing accuracy as $\rho$ increases and moves far from zero. That proves that Equation 12 cannot become zero for any non-negative value of $\rho$.

Equation 12 can be further simplified by restricting it to a selected subgroup of integers of interest, that is to say, to even integers (odd integers will be described later). In fact, $\Gamma\left(\frac{3+\rho}{2}\right)$ becomes a series of odd half integers, and $\Gamma\left(2+\frac{\rho}{2}\right)$ a series of integers. These factors can be approximated as

\begin{tabular}{|l|l|}
\hline$\Gamma\left(\frac{3+\rho}{2}\right)=\sqrt{\pi} \frac{(3+\rho-2) ! !}{2^{\frac{3+\rho-1}{2}}}=\sqrt{\pi} \frac{(\rho+1) ! !}{2^{\frac{\rho+2}{2}}} \quad \forall \rho \in 2 \square>-3$ & Equation 14 \\
\hline
\end{tabular}

and

$$
\Gamma\left(2+\frac{\rho}{2}\right)=\left(2+\frac{\rho}{2}-1\right) !=\left(\frac{\rho}{2}+1\right) ! \quad \forall \rho \in 2 \square>-3
$$

Equation 15

respectively, where !! represents the double factorial function; for odd values, this function can, 
in turn, be written as $m ! !=\prod_{k=0}^{\frac{m+1}{2}}(2 k-1)=m \cdot(m-2) \cdot(m-4) \cdot \ldots \cdot 3 \cdot 1$.

Thus, the ratio of Gamma functions can be rewritten as

\begin{tabular}{|l|l|}
\hline$\frac{\Gamma\left(\frac{3+\rho}{2}\right)}{\Gamma\left(2+\frac{\rho}{2}\right)}=\frac{(\rho+1) ! !}{\left(\frac{\rho}{2}+1\right) !} \cdot \sqrt{\pi} \cdot 2^{-\frac{\rho+2}{2}} \quad \forall \rho \in 2 \square>-3$ & Equation 16 \\
\hline
\end{tabular}

and thus Equation 12 can be circumscribed to the even integers of $\rho$ as

$$
\frac{k_{e q}}{k_{\rho+1}|a|^{\rho}}=\frac{(\rho+1) ! !}{\left(\frac{\rho}{2}+1\right) !} \cdot 2^{1-\frac{\rho+2}{2}} \quad \forall \rho \in 2 \square>-3
$$

Equation 17

Indeed, if one substitutes $\rho=2$ into Equation 17, the Duffing term is obtained as $k_{e q}=2^{-1} \cdot \frac{(3) ! !}{(2) !} k_{3} a^{2}=\frac{3}{4} k_{3} a^{2}$.

\subsection{The SDoF oscillator with generalised nonlinear damping}

The equation of motion (and related formulations) for thequadratic damping can begeneralised to the case with any value of the exponent $\eta$, similarly to what just presented for the cubic stiffness term. As it will be discussed later, it is also possible to expand the formulation to negative damping values, including also the Coulomb friction as a particular case of this generalised formulation.

The derivation of the generalised formulation is similar to the one for quadratic damping, except that here,

$$
\begin{array}{l|l|}
f_{d}(\dot{x})=c_{\eta+1} \dot{x}|\dot{x}|^{\eta} & \text { Equation } 18
\end{array}
$$

which becomes quadratic when $\eta=1$. As done in the case of the Duffing oscillator, let $x=a \sin (\Omega t-\varphi)$ be the trial output of the nonlinear equation of motion. If one considers $\varphi=0$, it can be seen that $f_{d}(\dot{x})=f_{d}(a \Omega \cos (\Omega t))$; this can be again approximated via first-order HB. $f_{d}(\dot{x})$ is also restricted to be an odd function. Since $f_{d}(\dot{x})=c_{e q} \dot{x}$ holds, after some steps, one finds 


$$
c_{e q}=\frac{1}{\Omega a \pi} \int_{0}^{2 \pi} f_{d}(\Omega a \cos (\theta)) \cos (\theta) d \theta
$$

with some further mathematical steps, it can be seen that

$$
c_{e q}=\frac{1}{\Omega a \pi} \int_{0}^{2 \pi} c_{\eta+1}|\Omega a \cos (\theta)|^{\eta} \Omega a \cos (\theta) \cos (\theta) d \theta=\frac{c_{\eta+1}}{\pi} \int_{0}^{2 \pi}|\Omega a \cos (\theta)|^{\eta} \cos ^{2}(\theta) d \theta
$$

similarly to the steps seen in Equation 6 and 7 , accounting for the $|\cdot|$ function leads to

$$
c_{e q}=4 \frac{|\Omega a|^{\eta} c_{\eta+1}}{\pi} \int_{0}^{1} \frac{\xi^{\eta+2}}{\sqrt{1-\xi^{2}}} d \xi
$$

Equation 21

defined $\forall \eta>-3$. Replicating the trigonometrical calculations as done to derive Equation 9 finally leads to

$$
c_{e q}=\left(\frac{\Gamma\left(\frac{3+\eta}{2}\right)}{\Gamma\left(2+\frac{\eta}{2}\right)}\right) \frac{2}{\sqrt{\pi}} c_{\eta+1}|\Omega a|^{\eta} \quad \forall \eta>-3
$$

Equation 22

One can notice easily that for $\eta=1, \Gamma\left(\frac{3+\eta}{2}\right)=\Gamma(2)=1$ and $\Gamma\left(2+\frac{\eta}{2}\right)=\Gamma\left(\frac{5}{2}\right)=\frac{3 \sqrt{\pi}}{4}$, which leads to $c_{e q}=\frac{8}{3 \pi} c_{2} \Omega a$ as expected. Departing from $c_{e q}$, one can define the equivalent damping ratio as well, by simply computing

$$
\zeta_{e q}=\frac{c_{e q}}{2 m \omega_{n}}=\left(\frac{\Gamma\left(\frac{3+\eta}{2}\right)}{\Gamma\left(2+\frac{\eta}{2}\right)}\right) \frac{c_{\eta+1}|\Omega a|^{\eta}}{m \sqrt{\pi} \omega_{n}} \quad \forall \eta>-3
$$

At this point, it is straightforward to plug $c_{e q}$ and/or $\zeta_{e q}$ into all the equations defined for the quadratic case.

The exact behaviour of $c_{e q}$ for a varying value of $\eta$ can be investigated similarly to what done before for $k_{e q}$. By analogy with Equation 12, it can be seen that the ratio $\frac{c_{e q}}{c_{\eta+1}|\Omega a|^{\eta}}$ is proportional to 


$$
g(\eta)=\frac{c_{e q}}{c_{\eta+1}|\Omega a|^{\eta}}=\left(\frac{\Gamma\left(\frac{3+\eta}{2}\right)}{\Gamma\left(2+\frac{\eta}{2}\right)}\right) \frac{2}{\sqrt{\pi}} \quad \forall \eta>-3
$$

which is illustrated in Figure 4.b. As seen for the equivalent stiffness (described by Equation 12 and portrayed for comparison in Figure 4.a), the value of the equivalent damping decreases with the increasing order and reaches zero for $\eta \rightarrow+\infty$ and $+\infty$ for $\eta \rightarrow-3$. While not explicitly portrayed in Figure 4.b, it can be seen that for the linear case where $\eta=0, g(\eta)$ reaches a unit value, exactly as for $g(\rho)$. In other words, this means that $c_{e q}=c_{1}|\Omega a|^{0}=c$ and the formulation reduces into its simple linear form.

[Figure 4 about here]

The formulation proposed in Equation 24 can be restricted to odd integers only, similarly to how Equation 12 was restricted to even integers in Equation 17. In this case, the numerator $\Gamma\left(\frac{3+\eta}{2}\right)$ becomes defined only over positive odd integers, for which $\Gamma(n)=(n-1)$ !, where $n=\frac{3+\eta}{2}$. Conversely, the denominator $\Gamma\left(2+\frac{\eta}{2}\right)$ becomes defined only over odd half-integers, and in this case $\Gamma\left(\frac{n^{\prime}}{2}\right)=\sqrt{\pi} \frac{\left(n^{\prime}-2\right) ! !}{2^{\frac{n^{\prime}-1}{2}}}$ where $\frac{n^{\prime}}{2}=2+\frac{\eta}{2}$. After some mathematical steps, this leads to

$$
c_{e q}=\frac{\left(\frac{\eta+1}{2}\right) !}{\pi(\eta+2) ! !} \cdot 2^{\frac{5+\eta}{2}} \cdot c_{\eta+1}|\Omega a|^{\eta} \quad \forall \eta \in 2 \square+1>-3
$$

As one can easily notice, if one replaces $\eta$ for $\rho, \frac{c_{e q}}{c_{\eta+1}|\Omega a|^{\eta}}$, described in Equation 24 and 
represented in Figure 4.b, shares the same functional form of $\frac{k_{e q}}{k_{\rho+1}|a|^{\rho}}$, as defined in Equation 12 and portrayed in Figure 4.a. Therefore, if one considers a general variable $\mu=\eta \vee \rho$, the two nonlinearities can be written with a unified non-dimensionalised formulation (for any odd or even integer larger than-3)

$$
f(\mu)=\left\{\begin{array}{l}
\frac{(\mu+1) ! !}{\left(\frac{\mu}{2}+1\right) !} \cdot 2^{1-\frac{\mu+2}{2}}, \forall \mu \in 2 \square>-3 \\
\frac{\left(\frac{\mu+1}{2}\right) !}{\pi(\mu+2) ! !} \cdot 2^{\frac{5+\mu}{2}}, \forall \mu \in 2 \square+1>-3
\end{array}\right.
$$

Equation 26

which is useful if one does not want to include non-integers powers in the formulations of Equation 4 or Equation 18. It should be remarked however that at moderate Reynold numbers, the nonlinear damping force was found to lie between the linear and the quadratic model, i.e. for $0<\eta<1$ ([32], Chapter 3.1). These findings may suggest the use of non-integer values in these cases.

The following remarks conclude the discussion about the definition of the generalised nonlinear damping.

\section{Remark 1: dimensional analysis}

Since $c_{e q} \propto c_{\eta+1} \cdot|\Omega a|^{\eta}$, knowing that the dimension of $c_{e q}$ and $|\Omega a|^{\eta}$ are, respectively, $\left[\frac{k g}{s}\right]$ and $\left[\frac{m^{\eta}}{s^{\eta}}\right]$, the dimensional analysis gives that the dimension of $c_{\eta+1}$ is $\left[\frac{\mathrm{kg} \cdot \mathrm{s}^{\eta-1}}{\mathrm{~m}^{\eta}}\right]$, which therefore is a function of only space and mass for the quadratic case (i.e. for $\eta=1$ ) and becomes instead dependent on time as well in any other case.

Remark 2: quadratic damping and Coulomb friction as particular cases.

It has been already proved how the quadratic damping fits the generalised formulation as a 
particular case for $\eta=1$. However, it is remarkable that Equation 22 and Equation 23 are not necessarily bounded to positive values of $\eta$. In particular, it is well-known that the Coulomb dry friction may be expressed as

$$
f_{d, \text { Coulomb }}(\dot{x})=c_{f} \cdot \operatorname{sgn}(\dot{x})=c_{f} \frac{\dot{x}}{|\dot{x}|}
$$

that is a specific case of Equation 18 for $\eta=-1$. The fact itself that the Nyquist plot of a quadratically-damped system and the Nyquist plot of a system with dry friction become distorted in complementary ways (elongating along the imaginary and real axis respectively; refer to the book of Worden and Tomlinson [7], Chapters 3.9.2 and 3.9.3, for further details) is a direct consequence of this dichotomy. It is known that in case of Coulomb dry friction, $c_{e q}$ equals $\frac{4 c_{f}}{\pi \Omega a}$, supported by the condition $a>\frac{4 c_{f}}{\pi}$ to avoid stick-slip motion (as intermittent motion invalidates this formulation). By imposing a negative unit value to $\eta$ in Equation 23, one obtains

\begin{tabular}{|l|l|}
\hline$\zeta_{e q}=\left(\frac{\Gamma\left(\frac{3+\eta}{2}\right)}{\Gamma\left(2+\frac{\eta}{2}\right)}\right) \frac{c_{\eta+1}|\Omega a|^{\eta}}{m \sqrt{\pi} \omega_{n}}=\frac{2 c_{0}}{m \pi \omega_{n} \Omega a}$ & Equation 28 \\
\hline
\end{tabular}

where $c_{0} \equiv c_{f}$. This is coincident with the expected value of $c_{e q}=\frac{4 c_{f}}{\pi \Omega a}$ when multiplied by the factor $2 m \omega_{n}$. The equivalent structural damping constant $h=\frac{4 c_{f}}{\pi}|a|$ can be derived from this generalised friction function as well. It is also important to notice how for any $\eta<0$, Equation 18 becomes a function of the inverse of the displacement amplitude. This is observed in Coulomb friction, where it is well-known that the nonlinearity increases for decreasing input energy, becoming almost linear at (relatively) for high input amplitudes. On the other hand, the effects of the quadratic damping increase for an increasing displacement.

\subsection{The SDoF oscillator with combined generalised nonlinearities}


Having defined the generalised functions of nonlinear stiffness and damping, it is possible to replace the Duffing and/or the quadratic damping term with them. Two specific cases have been investigated here. Firstly, the assumption of cubic stiffness was kept while relaxing the definition of nonlinear damping to accommodate any possible exponent. This resulted in the nonlinear terms $k_{3} x^{3}+c_{\eta+1} \dot{x}|\dot{x}|^{\eta}$. This was motivated by the specific experimental dataset analysed, where (as it will be shown later in Section 4) the error in frequency due to using the Duffing term was much lower than the error in amplitude due to using the quadratic damping.

One can then derive the modulation equations, the eigenvalues, the corresponding stability criterion, and the steady state solution in the frequency domain with the same mathematical steps as for the other models.

The model with both generalised nonlinear damping and stiffness is described next. In this case, the nonlinearities are governed by the two exponents $\eta$ and $\rho$ and by the coefficients of their respective terms $\left(c_{\eta+1}\right.$ and $\left.k_{\rho+1}\right)$. The equation of motion becomes

$$
m \ddot{x}+c \dot{x}+k x+c_{\eta+1} \dot{x}|\dot{x}|^{\eta}+k_{\rho+1} x|x|^{\rho}=F \cos (\Omega t)
$$

The resulting steady state solution in the frequency domain is very similar to Equation 11, that is to say,

$$
\frac{\Omega}{\omega_{n}}=1+\frac{1}{2 m \omega_{n}^{2}} k_{e q} \pm \sqrt{\frac{\gamma^{2}}{\left(2 \omega_{n}^{2}\right)^{2} a^{2}}-\left(\zeta+\zeta_{e q}\right)^{2}}
$$

Equation

i.e. only adding the term $\zeta_{e q}$ as defined in Equation 23. As before, it is then possible to derive the modulation equations and all the other related formulations.

To recapitulate the proposed analytical models, these are summarised in Table 4 and compared to their classic counterparts.

[Table 4 about here]

\section{Fitting procedure and results}




\subsection{The fitting procedure}

This whole discussion aims to calibrate the parameters $\theta$ of the investigated system. These are a mixture of linear and nonlinear terms of damping and stiffness, i.e. $\theta=\left\{\boldsymbol{\theta}_{L I N}, \boldsymbol{\theta}_{\text {NONLIN }}\right\}$. The former group is made up by the classic terms $\theta_{L I N}=\{m, c, k\}$. The latter one depends on the specific case considered among the ones described in the previous Section 3. In the case of the completely generalised model, these are $\theta_{\text {NONLIN }}=\left\{\eta, c_{\eta+1}, \rho, k_{\rho+1}\right\}$ and the whole set of parameters becomes $\boldsymbol{\theta}_{\eta, \rho}=\left\{m, c, k, \eta, c_{\eta+1}, \rho, k_{\rho+1}\right\}$. For the case with mixed Duffing nonlinear stiffness and generalised nonlinear damping, these terms are reduced to $\theta_{\eta, 2}=\left\{m, c, k, \eta, c_{\eta+1}, k_{3}\right\}$ being $\rho=2$. In the classic cubic stiffness-quadratic damping model, these are further reduced to $\theta_{1,2}=\left\{m, c, k, c_{2}, k_{3}\right\}$, since this model corresponds to imposing $\eta=1$ and $\rho=2$ to the generalised formulation.

The fitting is performed separately on the data collected from the unloaded and the sensor-loaded structure; henceforth, only five input levels are available for each case. Nevertheless, the parameters of the linear system $\theta_{L I N}$ can be estimated from the response to low-level excitations, where the structure behaves linearly, as explained in Section 2.3; this is quite often done in common practice (see e.g. Reference [41]). The pre-existing linear terms are unaffected by the additional nonlinearities, which are directly summable, and considered independent from the amplitude of the input. Both options (estimating separately the linear and nonlinear terms or optimising over all the parameters at once) were tested for the fully generalised model, with inconsequential differences (see Section 4.4).

The strategy pursued was to fit the steady state amplitude responses over the analytical models, considering the output displacements and the input accelerations. The transient phase at the beginning of each new frequency step was discarded, resorting to the data portrayed in dark blue in Figure 2. The optimisation of the parameters can be performed by minimising the error between the analytical formulation and the experimental data. For five levels of amplitude in input and the response in the frequency domain discretised in 200 frequency steps, that means 


$$
\boldsymbol{\theta}_{\text {NONLIN }^{*}}=\left.\underset{\Theta_{\text {NONLIN }}}{\arg \min } \sum_{A=1}^{5} \sum_{k=1}^{200}\left|\left(\Omega_{k}, \frac{\hat{X}_{k}}{\left|\gamma_{A}\right|}\right)_{A}-\left(\Omega_{k}, \frac{X_{k}}{\left|\gamma_{A}\right|}\right)_{A}\right|\right|^{2}
$$

Equation 31

being $\hat{X}_{k}$ the recorded steady state output displacement amplitude for the $k$-th step, coupled with

the corresponding driving frequency $\Omega_{k}$ at the $A$-th energy level of the input; $X_{k}$ indicates the displacement amplitude of the model response for the same $\Omega_{k}$ and $\left|\gamma_{A}\right|$ is the amplitude of the input acceleration for the corresponding $A$-th energy level. The MatLab ${ }^{\circledR}$ Optimization Toolbox ${ }^{\mathrm{TM}}$ algorithm fmincon( ) was used ${ }^{1}$. The initial guess was chosen close enough to the estimated global minimum so that the algorithm fell directly into its capture basin. This was also confirmed by further investigations using Multistart( ) and GlobalSearch ( $)^{2}$ from the MatLab Global Optimization Toolbox $^{\mathrm{TM}}$. This further study verified the existence of many local minima (at least 17 for both the loaded and unloaded conditions), but all of them characterised by physically meaningless parameters (i.e. unrealistically higher exponents, with $\eta$ and/or $\rho$ always higher than 50).

It must be stated that the fitting can be performed in the time domain as well; however, in this specific application, it is much more efficient to perform it on the frequency domain. Firstly, the steady state response was already available with the data pre-processed at the machine level, with the transient discarded at any frequency step. Moreover, the steady state frequency responses defined by Equation 30 can be rapidly defined analytically over the 200 frequency steps of interest, while fitting over time would require solving the system of differential equations derived from a state space representation of Equation 29. This becomes computationally expensive for very long acquisitions as here. For instance, the longest recording accounts for 8,752,000 timesteps corresponding to slightly more than 1 hour 11 minutes of recording. The input signal was subsampled from its original sampling frequency of $2048 \mathrm{~Hz}$ to $64 \mathrm{~Hz}$ without losing frequency and amplitude information (further subsampling was found to lose information on the amplitude of the response). Even so, to simulate the system response to the 273500-datapoint-long reduced input, the

\footnotetext{
${ }^{1}$ https://it.mathworks.com/help/optim/ug/fmincon.html (last visited 22nd June 2020).

${ }^{2}$ https://it.mathworks.com/help/gads/how-globalsearch-and-multistart-work.html (last visited 22nd June 2020).
} 
computational time (up to more than 40 minutes per simulation) was too long to make it efficient for the minimisation task.

\section{2. $\quad$ State of the art: the quadratic damping - cubic stiffness model}

The classic models with cubic (Duffing) stiffness only and quadratic damping only were firstly tested on the experimental data. In the first case, the cumulative error in frequency was relatively low. However, a nonlinear damping term was clearly needed to adjust the amplitude response.

The model with quadratic damping only performed very poorly in this sense. Apart from the frequency error, due to the absence of a term governing the frequency shift of the phase response, the error in peak amplitude was always unacceptably high regardless of the value of $c_{2}$. Specifically, the error arises because of poor fitting on the higher input energy levels, or due to poor fitting at lower amplitudes. Generally, any coefficient value will return a model that is or underdamped for $\gamma=0.82$ and $1.24 \mathrm{~m} / \mathrm{s}^{2}(\mathrm{P}-\mathrm{P})$ or overdamped for $\gamma=1.62$ and $2.00 \mathrm{~m} / \mathrm{s}^{2}$.

These preliminary studies were used to test separately the single parts which make up the classic mixed model. The numerical simulations for the combined Duffing stiffness and quadratic damping model, carried out from the modulation equations, are represented in Figure 5. In the Case a (Figure 5.a), the model is well fitted over the lower energy levels, but severely underdamped at the higher input amplitudes. Vice versa, in Case b (Figure 5.b), the model parameters are best fitted for the higher levels but at the cost of being overdamped at low input amplitudes. The obtained parameters are reported in Table 5. The global error, computed cumulatively at all the five energy levels, is lower in the second case (please notice that in Figure 5.a the y-axis limit had to be increased to accommodate the excessively high estimated amplitude). Yet, the model is evidently unfit in both cases.

[Figure 5 about here.]

[ Table 5 about here.]

\section{3. $\quad$ The generalised nonlinear damping - cubic stiffness model}


To test the proposed generalised formulations, the following analyses where performed, considering the two variants described in the previous Sections: the generalised nonlinear damping model retaining the cubic stiffness and the fully generalised nonlinear model. Moreover, integer and non-integer values of $\eta$ and $\rho$ were both tested in all cases.

The resulting parameters of the model with Duffing nonlinear stiffness and generalised nonlinear damping can be found in Table 6. Figure 6.a and Figure 6.b depict, respectively, the numerical simulations carried out from the modulation equations with the Duffing stiffness term and (in this order) integer and non-integer values of $\eta$.

[Figure 6 about here.]

[Table 6 about here.]

\subsection{The generalised nonlinear damping and stiffness model}

The resulting parameters for the model with mixed generalised nonlinear stiffness and damping are enlisted in Table 7. The results are shown for the case with the linear parameters pre-estimated from the linear response (i.e., optimising only the full set of the nonlinear parameters $\theta_{\text {NONLIN }}$ ) and for the case with all model parameters (linear and nonlinear) estimated from the nonlinear response only. The results were found to be very similar. The loaded and unloaded models with pre-estimated linear parameters and integer values of $\eta$ and $\rho$ are graphically displayed (through calibrated modulation equations) in Figure 7.a. The outcome of the same optimisation letting the two exponents free to acquire any non-integer values are reported in Figure 7.b. As one can directly infer, these results depicted in Figure 7 are almost coincident in terms of effective damping to the ones reported in Figure 6. They are distinguished by a slightly better fitting in terms of the hardening behaviour yet at the expenses of a rater more complicated model. It is left to the engineer's choice if the trade-off between the greater accuracy and the additional complication of further adding a parameter to be estimated is cost-efficient, also depending on the intended aim. The quantitative values of the amplitude and frequency at phase quadrature are reported in Table 8 and compared to 
the experimental truth. As it can be seen, the error is never too large neither in the amplitude nor in the frequency domain. Frequency-wise, the generalised nonlinear damping and stiffness model shows some improvements respect to the mixed generalised nonlinear damping - Duffing stiffness model, where the error in the estimated frequency (with properly calibrated coefficients) was always one order of magnitude larger.

[Figure 7 about here.]

[Table 7 about here.]

[Table 8 about here.]

\subsection{Validation in the time-frequency domain}

To conclude this study, the proposed models have been validated in the time-frequency domain, comparing the experimental output recordings to the results of the numerical simulations run using the proposed model. Specifically, the results from the model with generalised nonlinear stiffness and damping for integer values of $\rho$ and $\eta$ are here discussed.

Considering $y(t)=\dot{x}(t)$ and $\dot{y}(t)=\ddot{x}(t)$, the equations of motion in Equation 29 can be written in the state-space representation as

$$
\left\{\begin{array}{l}
\dot{y}=-\delta \dot{x}-\alpha x-\frac{k_{\rho+1}}{m} x|x|^{\rho}-\frac{c_{\eta+1}}{m} \dot{x}|\dot{x}|^{\eta}+\gamma \\
y=\dot{x}
\end{array}\right.
$$

with the estimated linear and nonlinear parameters $m, c, k, k_{\rho}, \rho, c_{\eta}, \eta$.

This system of differential equations has been fed with the experimentally-recorded time histories of the input acceleration $\gamma$; the resulting output has been then compared to the recorded output through their respective spectrograms (reported in Figure 8). These were computed via Short-Time Fourier Transforms (STFTs), with L-point symmetric Hamming windows (L being set to one- 
fifteenth of the total data points in the recording) and 50\% overlap between contiguous sections. Please notice that the step duration is not constant, nor it was equal for the different levels of the input amplitude, due to the automatic procedure of the stepped sine. Figure 8.a and Figure 8.b compare the 3-D view of the spectrogram of the recorded data and the calibrated model for the unloaded and loaded cases (in this order). Due to space limitations, only the first, third, and last input levels are portrayed. The model catches overall well the global dynamic behaviour of the structure in the time-frequency domain. Negligible differences were encountered when considering non-integer values of $\rho$ and $\eta$. The maximum differences in the time-frequency plane between the simulations and the recorded data are indicated in Figure 8 as well. These have been calculated as

$$
\max \left(\frac{\left|s_{\text {exp }}(f, t)-s_{\text {num }}(f, t)\right|}{\left|s_{\text {exp }}(f, t)\right|}\right) \cdot 100
$$

Equation 33

where $s_{\exp }(f, t)$ is the value of the spectrogram as identified from the experimental recordings at the frequency $f$ and instant $t$, while $s_{\text {num }}(f, t)$ is the same value as identified from the numerically simulated output.

[Figure 8 about here.]

\subsection{Discussion}

The main results for the specific case study here investigated can be enlisted as follow:

- The quadratic damping model, usually proposed in the scientific literature to model the airinduced drag of fast-moving beam-like structures, proved to be unfit to represent adequately the velocity-dependent nonlinearities. This may be due to the tapered shape of the plate-like spar or to other unaccounted sources of viscous nonlinear damping. Higher-order models (i.e. with $\eta>1$ ) proved to fit the experimental data with much higher accuracy.

- The cubic stiffness model (i.e. the Duffing model) proved to fit the hardening behaviour of the structure subject to increasing input energy levels with an error margin which may be acceptable for 
some specific uses. Nevertheless, higher-order nonlinear stiffness provided some quantifiable improvements (but at the cost of adding a further parameter to be calibrated).

- As a result, the proposed SDoF nonlinear Reduced Order Model was able to fit with good accuracy the steady state response of the mechanical system under scrutiny.

- Fitting the experimentally defined amplitude response functions takes only a few seconds with a non-optimised code, providing an immediate calibration of the nonlinear parameters. Thus, its computational time and effort are generally (much) lower than fitting the simulated output time series.

- The calibration must be performed cautiously as it may fall in local minima if the starting values are far from the expected results. This issue is particularly relevant for the two exponents $\rho$ and $\eta$, as any change can produce vastly different outcomes.

\section{Conclusions}

In standard practice, whenever the structure of interest cannot be approximated to a linear model, specific parametric models are generally applied, incorporating all the available knowledge about its structural nonlinearities. This approach, however, may lack adaptability. In this work, the classic analytical model - made up by the Duffing stiffness and the quadratic damping - was generalised, obtaining a model which is still simple but more versatile. A power-law formulation was applied, for any arbitrary values of the exponents $\rho$ and $\eta$. Two versions have been provided: with $\rho$ and $\eta$ free to assume any real value and with them restricted to integers, with a dual formulation for even and odd values. The corresponding equivalent stiffness and damping have been analytically defined. It was found that both the Coulomb dry friction model and the quadratic damping model come as particular cases of the generalised nonlinear damping formulation.

The obtained formulations may be further complicated if needed to adapt for specific aims. In the basic formulation reported here, they were found able to describe the flap-wise nonlinear large 
oscillations of a cantilevered aluminium spar with good accuracy at different levels of the input amplitude. That led to a single DoF model with multiple nonlinear terms. This model can be fitted from frequency domain data with a very low computational effort; it is also fully inspectable while leaving the exponents $\rho$ and $\eta$ in the nonlinear stiffness and damping terms completely free to adapt, thus not constrained by any strong assumption on the actual physics of the problem. This allows a significant versatility of the model, which may be applied to other nonlinear systems from different fields of application.

\section{Acknowledgements}

The Authors would like to thank Prof. Keith Worden for his advice and useful recommendations, Dr Ivan Petrunin and Dr Alessandro Pontillo for their assistance with the experimental setup, and Dr Mudassir Lone for kindly providing the wing spar.

\section{References}

[1] G. Kerschen, M. Peeters, J.C. Golinval, C. Stéphan, Nonlinear Modal Analysis of a Full-Scale Aircraft, J. Aircr. 50 (2013) 1409-1419. https://doi.org/10.2514/1.Co31918.

[2] B. Peeters, H. Climent, R. de Diego, J. de Alba, J. Rodriguez Ahlquist, J. Martinez Carreño, W. Hendricx, A. Rega, G. García, J. Deweer, J. Debille, Modern solutions for Ground Vibration Testing of large aircraft, J. Sound Vib. 43 (2009).

[3] D. Laxalde, F. Thouverez, Complex non-linear modal analysis for mechanical systems: Application to turbomachinery bladings with friction interfaces, J. Sound Vib. 322 (2009) 1009-1025. https://doi.org/10.1016/j.jsv.2008.11.044.

[4] N. Pugno, C. Surace, R. Ruotolo, Evaluation of the Non-linear Dynamic Response to Harmonic Excitation of a Beam with Several Breathing Cracks, J. Sound Vib. (2000) 749-762. https://doi.org/10.1006/jsvi.2000.2980. 
[5] G. Kerschen, K. Worden, A.F. Vakakis, J.-C. Golinval, Past, present and future of nonlinear system identification in structural dynamics, Mech. Syst. Signal Process. 20 (2006) 505-592. https://doi.org/10.1016/J.YMSSP.2005.04.008.

[6] J.P. Noël, G. Kerschen, Nonlinear system identification in structural dynamics: 10 more years of progress, Mech. Syst. Signal Process. 83 (2017) 2-35. https://doi.org/10.1016/J.YMSSP.2016.07.020.

[7] K. Worden, G.R. Tomlinson, Nonlinearity in structural dynamics : detection, identification, and modelling, Institute of Physics Publishing, 2001.

[8] J. Schoukens, R. Pintelon, T. Dobrowiecki, Y. Rolain, Identification of linear systems with nonlinear distortions, Automatica. 41 (2005) 491-504. https://doi.org/10.1016/J.AUTOMATICA.2004.10.004.

[9] T.P. Dobrowiecki, J. Schoukens, Practical choices in the FRF measurement in presence of nonlinear distortions, in: IMTC/99. Proc. 16th IEEE Instrum. Meas. Technol. Conf. (Cat. No.99CH36309), IEEE, n.d.: pp. 922-927. https://doi.org/10.1109/IMTC.1999.776998.

[10] E. Balmès, J. Wright, GARTEUR Group on Ground Vibration Testing. Results from the Test of a Single Structure by 12 Laboratories in Europe, 1996.

[11] C. Surace, K. Worden, G.R. Tomlinson, On the non-linear characteristics of automotive shock absorbers, Proc. Inst. Mech. Eng. Part D J. Automob. Eng. 206 (1992) 3-16.

[12] C. Surace, K. Worden, G.R. Tomlinson, An improved nonlinear model for an automotive shock absorber, Nonlinear Dyn. 3 (1992) 413-429. https://doi.org/10.1007/BFooo45646.

[13] F. Cottone, H. Vocca, L. Gammaitoni, Nonlinear Energy Harvesting, Phys. Rev. Lett. 102 (2008). https://doi.org/10.1103/PhysRevLett.102.080601.

[14] Y. Ren, T.M. Lim, M.K. Lim, Identification of properties of nonlinear joints using dynamic test data, J. Vib. Acoust. Trans. ASME. 120 (1998) 324-330. https://doi.org/10.1115/1.2893834.

[15] G. Duffing, Erzwungene Schwingungen bei Veranderlicher Eigenfrequenz (Forced Oscillations in the 
Presence of Variable Eigenfrequencies), F. Vieweg Sohn. (1918) 41-42.

[16] A.A. Muravyov, Determination of Nonlinear Stiffness Coefficients for Finite Element Models with Application to the Random Vibration Problem, 1999.

[17] J.L. Huang, R.K.L. Su, Y.Y. Lee, S.H. Chen, Nonlinear vibration of a curved beam under uniform base harmonic excitation with quadratic and cubic nonlinearities, J. Sound Vib. 330 (2011) 5151-5164. https://doi.org/10.1016/j.jsv.2011.05.023.

[18] B.R. Smith, The quadratically damped oscillator: A case study of a non-linear equation of motion, Am. J. Phys. 80 (2012) 816-824. https://doi.org/10.1119/1.4729440.

[19] F. Cao, Y. Ge, Air-induced nonlinear damping and added mass of vertically vibrating bridge deck section models under zero wind speed, J. Wind Eng. Ind. Aerodyn. 169 (2017) 217-231. https://doi.org/10.1016/j.jweia.2017.07.022.

[20] W. Zhang, G. Meng, Nonlinear dynamical system of micro-cantilever under combined parametric and forcing excitations in MEMS, Sensors Actuators, A Phys. 119 (2005) 291-299. https://doi.org/10.1016/j.sna.2004.09.025.

[21] A. Eichler, J. Moser, J. Chaste, M. Zdrojek, I. Wilson-Rae, A. Bachtold, Nonlinear damping in mechanical resonators made from carbon nanotubes and graphene, Nat. Nanotechnol. 6 (2011) 339-342. https://doi.org/10.1038/nnano.2011.71.

[22] H. Sumali, T.G. Carne, Air-Drag Damping on Micro-Cantilever Beams, 2008.

[23] M. Amabili, Nonlinear damping in large-amplitude vibrations: modelling and experiments, Nonlinear Dyn. 93 (2018) 5-18. https://doi.org/10.1007/s11071-017-3889-z.

[24] A. Pontillo, D. Hayes, G. Dussart, G. Lopez, M. Carrizalez, S. Yusuf, M. Lone, Flexible high aspect ratio wing: Low cost experimental model and computational framework, in: AIAA Atmos. Flight Mech. Conf. 2018, American Institute of Aeronautics and Astronautics Inc, AIAA, 2018. https://doi.org/10.2514/6.2018-1014. 
[25] M. Civera, L. Zanotti Fragonara, C. Surace, Video Processing Techniques for the Contactless Investigation of Large Oscillations, J. Phys. Conf. Ser. 1249 (2019).

[26] M. Civera, L. Zanotti Fragonara, C. Surace, Using Video Processing for the Full-Field Identification of Backbone Curves in Case of Large Vibrations, Sensors. 19 (2019) 2345. https://doi.org/10.3390/s19102345.

[27] M. Civera, M. Ferraris, R. Ceravolo, C. Surace, R. Betti, The Teager-Kaiser Energy Cepstral Coefficients as an Effective Structural Health Monitoring Tool, Appl. Sci. 9 (2019) 5064. https://doi.org/10.3390/app9235064.

[28] M. Civera, C. Surace, L.Z. Fragonara, A Computer Vision-based Approach for Non-contact Modal Analysis and Finite Element Model Updating, in: EWSHM2020 (In Publ., 2020.

[29] J.J. Ryan, J.T. Bosworth, J.J. Burken, P.M. Suh, Current and Future Research in Active Control of Lightweight, Flexible Structures Using the X-56 Aircraft, in: 52nd Aerosp. Sci. Meet., 2014.

[30] B.P. Iii, S.R. Cole, G.D. Miller, A Summary of the Active Flexible Wing Program, J. Aircr. 32 (1995) 10-15.

[31] T.E. Noll, F.E. Eastep, Active flexible wing program, J. Aircr. 32 (1995) 9. https://doi.org/10.2514/3.56918.

[32] A.H. Nayfeh, D.T. Mook, Nonlinear oscillations, Wiley \& Sons, 1979.

[33] DataPhysics, SignalCalc Dynamic Signal Analyzer User Manual, Chapter 20, (n.d.). https://nvtgroup.app.box.com/s/ym4h8mdo7pi78hfx9ibt83qo2cioifuf.

[34] S. Grivet-Talocia, B. Gustavsen, Passive Macromodeling: Theory and Applications, Wiley, 2016.

[35] B. Gustavsen, A. Semlyen, Rational approximation of frequency domain responses by vector fitting, IEEE Trans. Power Deliv. 14 (1999) 1052-1059. https://doi.org/10.1109/61.772353.

[36] D. Deschrijver, M. Mrozowski, T. Dhaene, D. De Zutter, Macromodeling of multiport systems using a fast implementation of the vector fitting method, IEEE Microw. Wirel. Components Lett. 18 (2008) 383-385. https://doi.org/10.1109/LMWC.2008.922585.

[37] M. Civera, G. Calamai, L. Zanotti Fragonara, Experimental Modal Analysis of Structural Systems by Using 
the Fast Relaxed Vector Fitting Method, Struct. Control Heal. Monit. (2020).

[38] K. Mam, M. Peigney, D. Siegert, Finite strain effects in piezoelectric energy harvesters under direct and parametric excitations, J. Sound Vib. 389 (2017) 411-437. https://doi.org/10.1016/j.jsv.2016.11.022.

[39] P. Malatkar, A.H. Nayfeh, A Parametric Identification Technique for Single-Degree-of-Freedom Weakly Nonlinear Systems with Cubic Nonlinearities, Modal Anal. 9 (2003) 317-336.

https://doi.org/10.1177/107754603030754.

[40] M.M. Kamel, Y.A. Amer, Response of Parametrically Excited One Degree of Freedom System With Nonlinear Damping and Stiffness, Phys. Scr. 66 (2002) 410-416.

https://doi.org/10.1238/physica.regular.066aoo410.

[41] R. Ceravolo, A. De Marchi, E. Pinotti, C. Surace, L. Zanotti Fragonara, Measurement of weak non-linear response of Kevlar@ fibre damaged by UV exposure, Compos. Struct. 184 (2018) 807-813. https://doi.org/10.1016/j.compstruct.2017.10.056. 


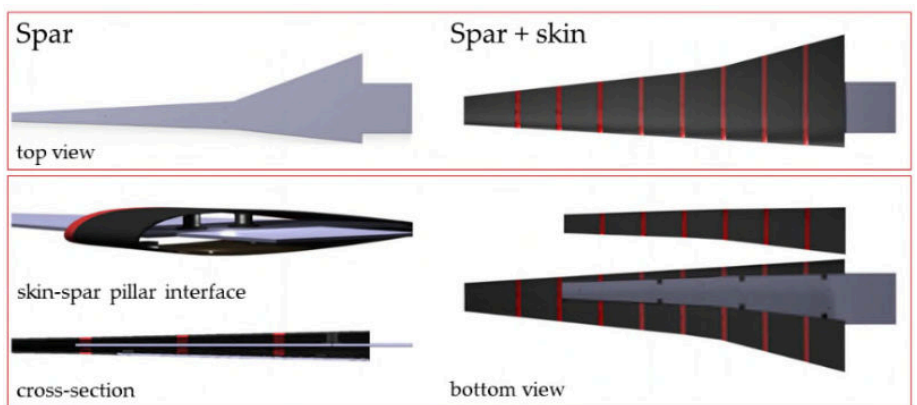

(a)

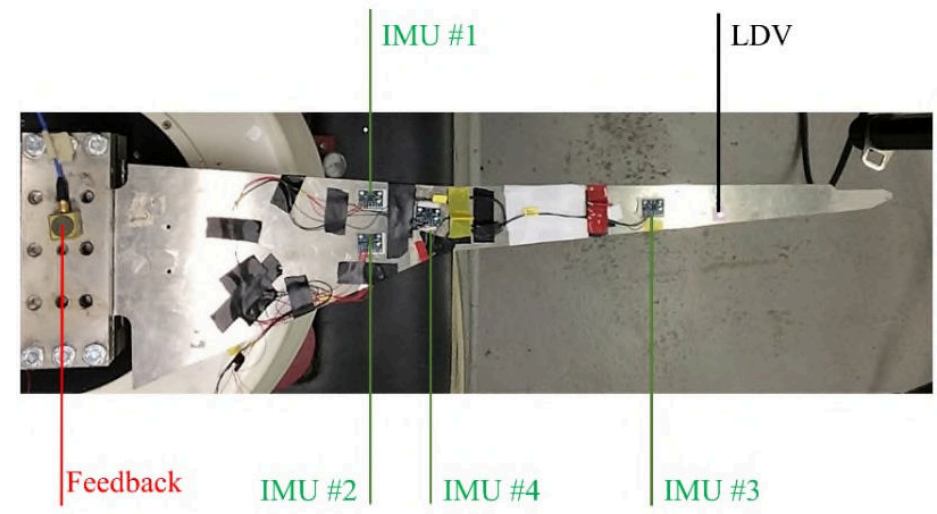

(c)

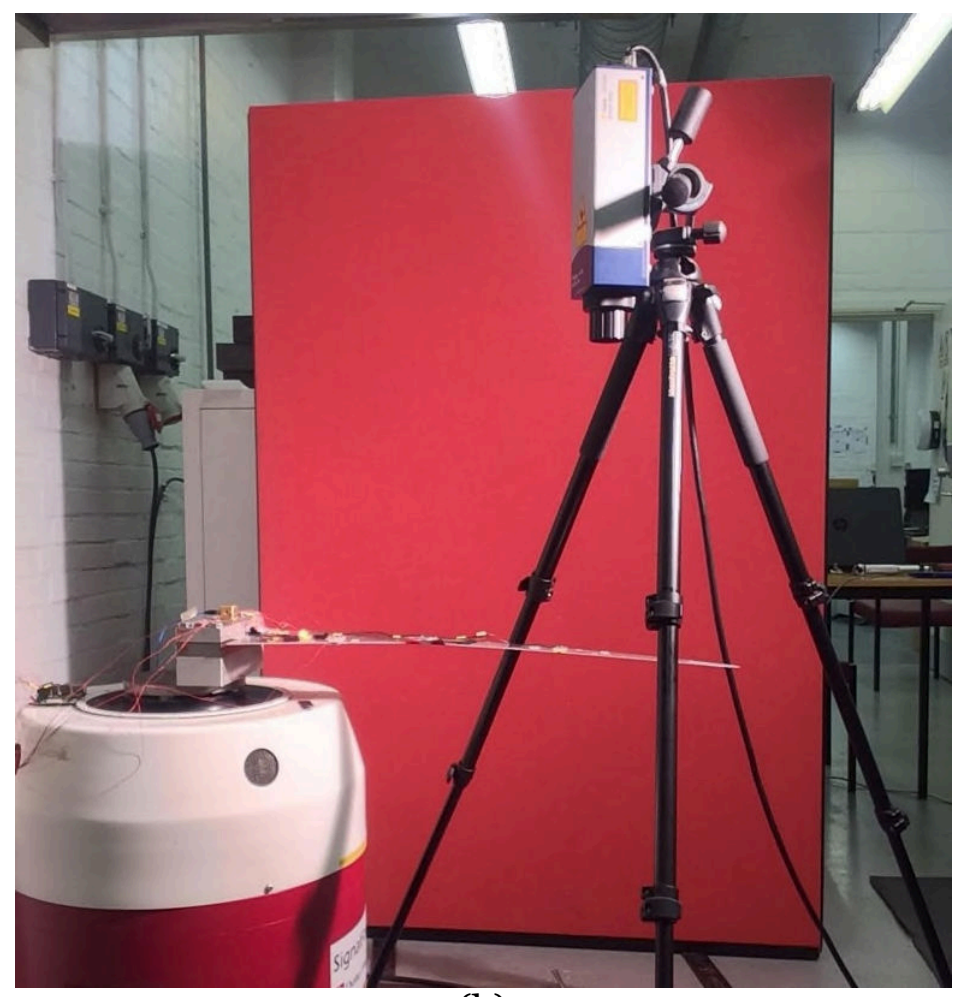

(b)

Figure 1. (a) the schematics of the wing and its spar (adapted from [26]). (b) the experimental setup (c) The location of the four IMUs for the sensor-loaded configuration 


\section{Sensor-loaded Configuration}
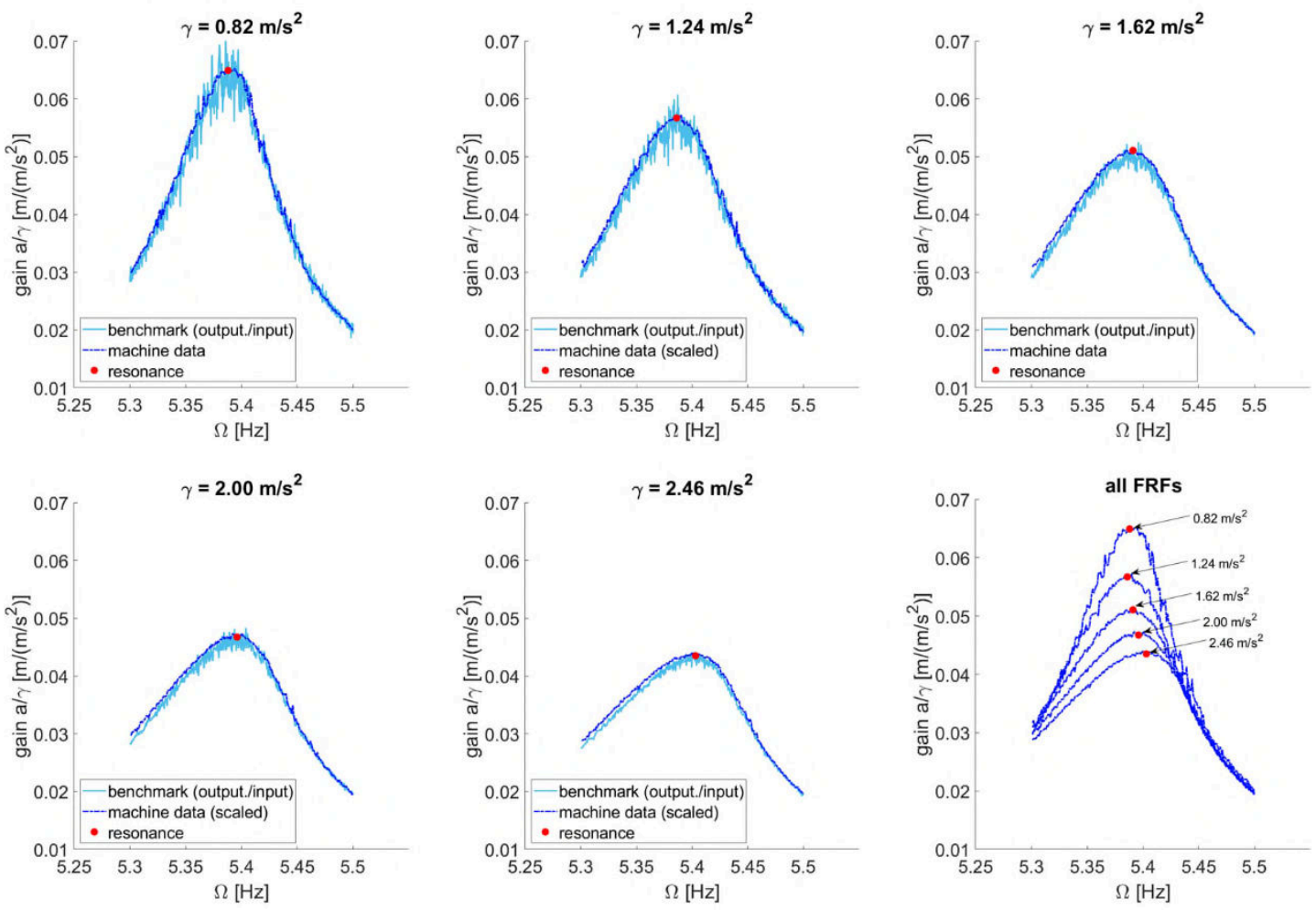

(a)

\section{Unloaded Configuration}
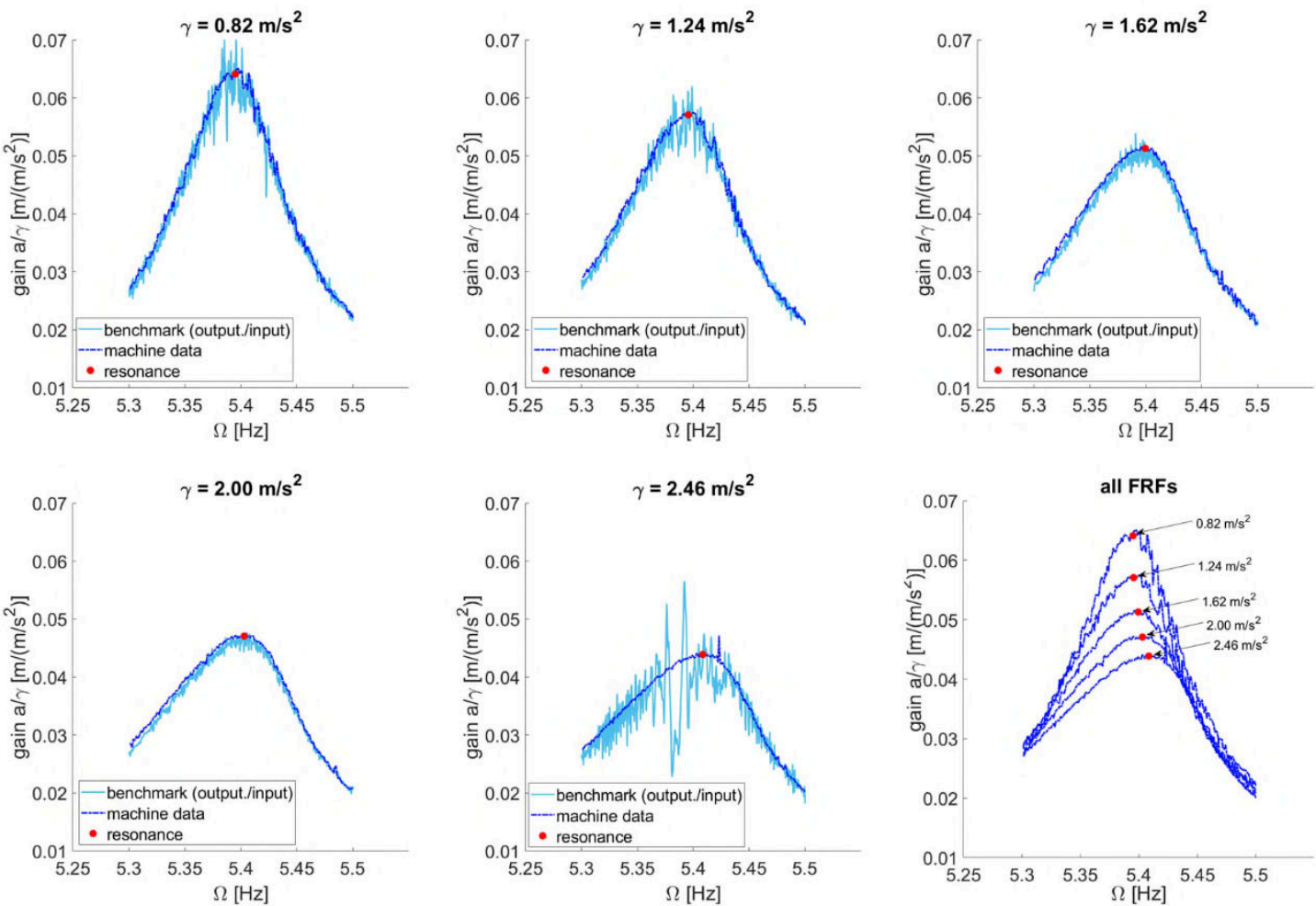

(b)

Figure 2. Amplitude response functions (acceleration in input, displacement in output) as directly returned from the software (dark blue lines) and as calculated by dividing the output Fourier spectra by the input Fourier spectra (light blue lines). (a) Unloaded case, (b) sensor-loaded case. All values are expressed in terms of peak-to-peak $(P-P)$ amplitude. 


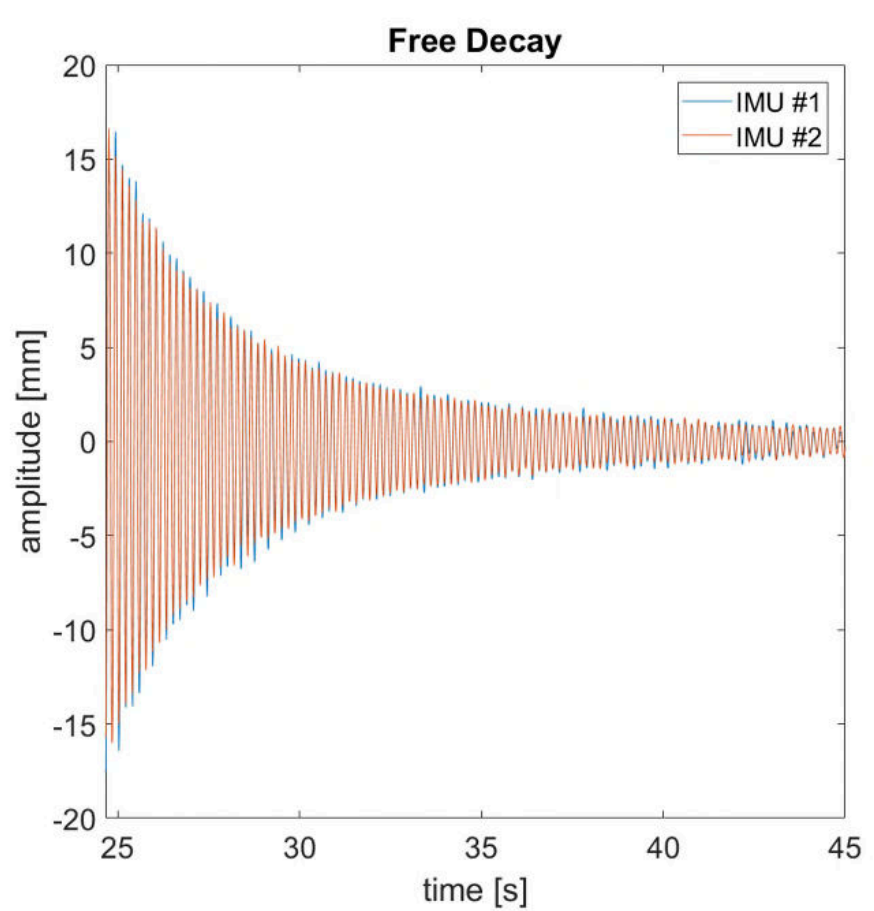

(a)

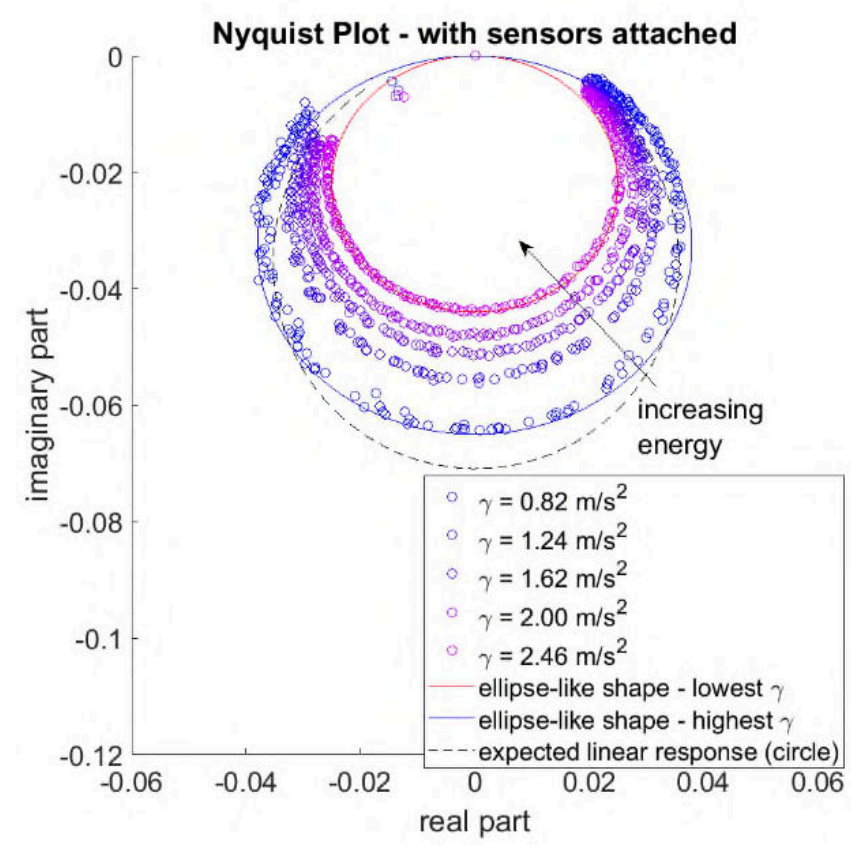

(c)

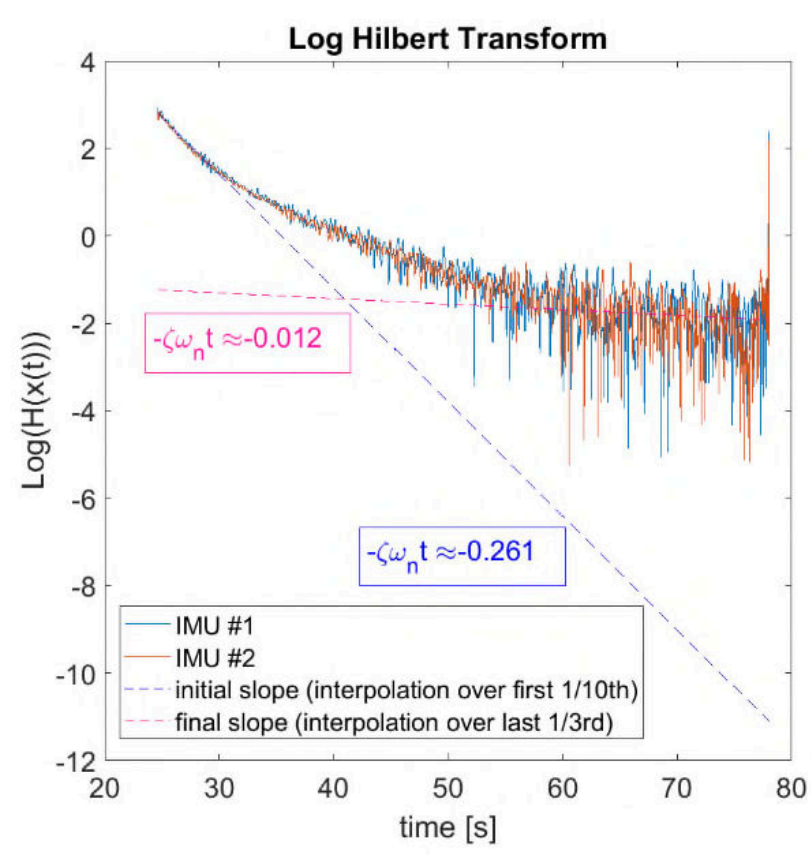

(b)

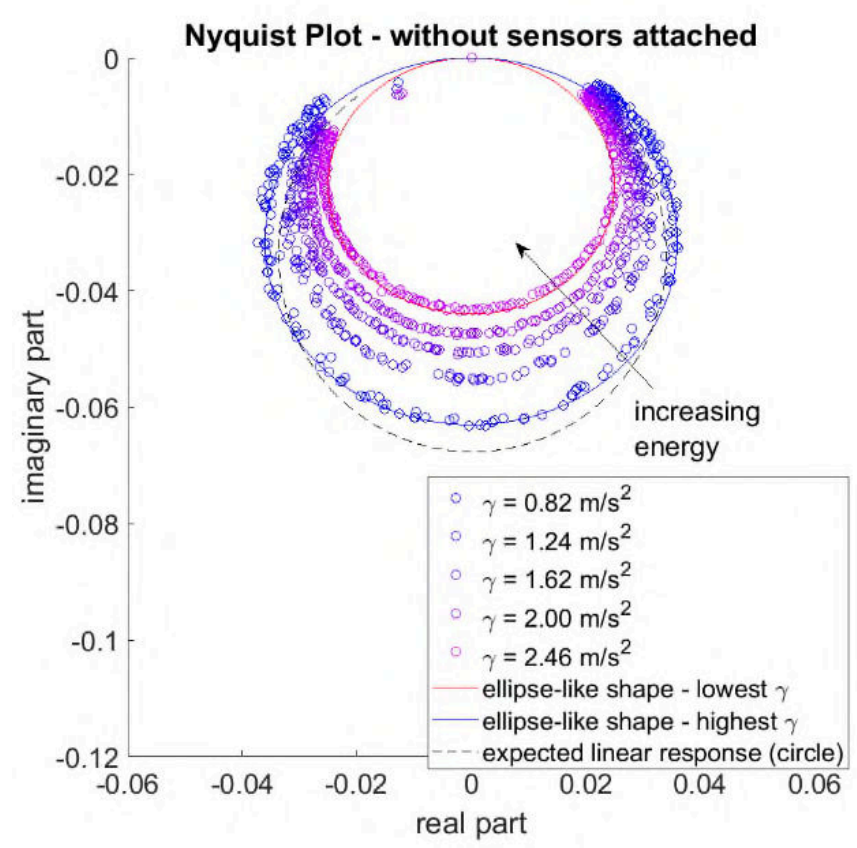

(d)

Figure 3. Top row: (a) recorded free decay (from [26]) and (b) log-Hilbert of the free oscillations highlighting nonlinearities in the damping. Bottom row: Nyquist plots: (a) configuration with sensors attached and (b) without sensors attached 


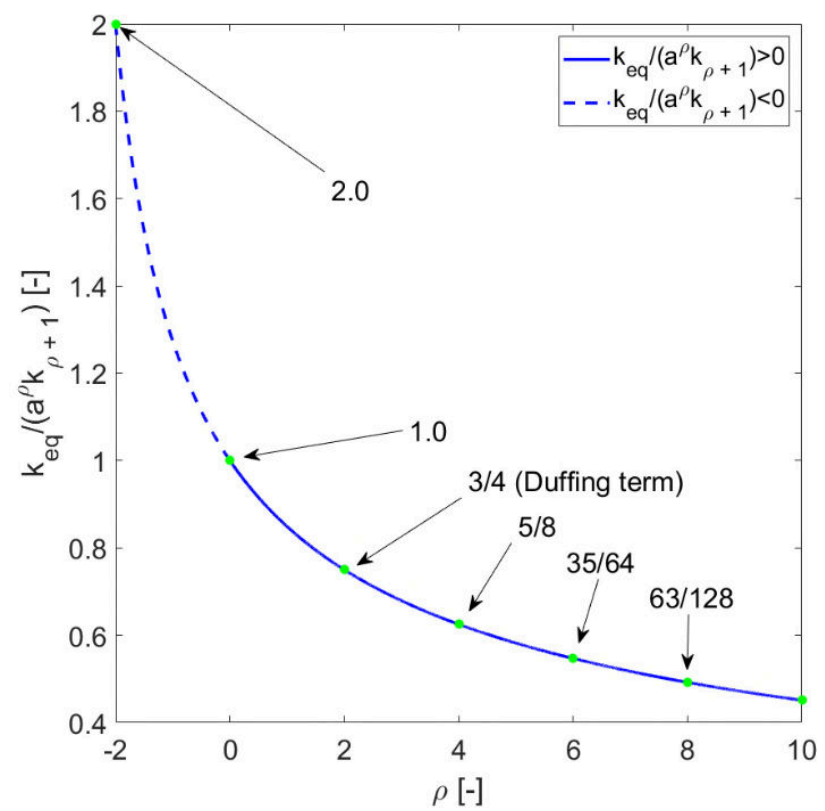

(a)

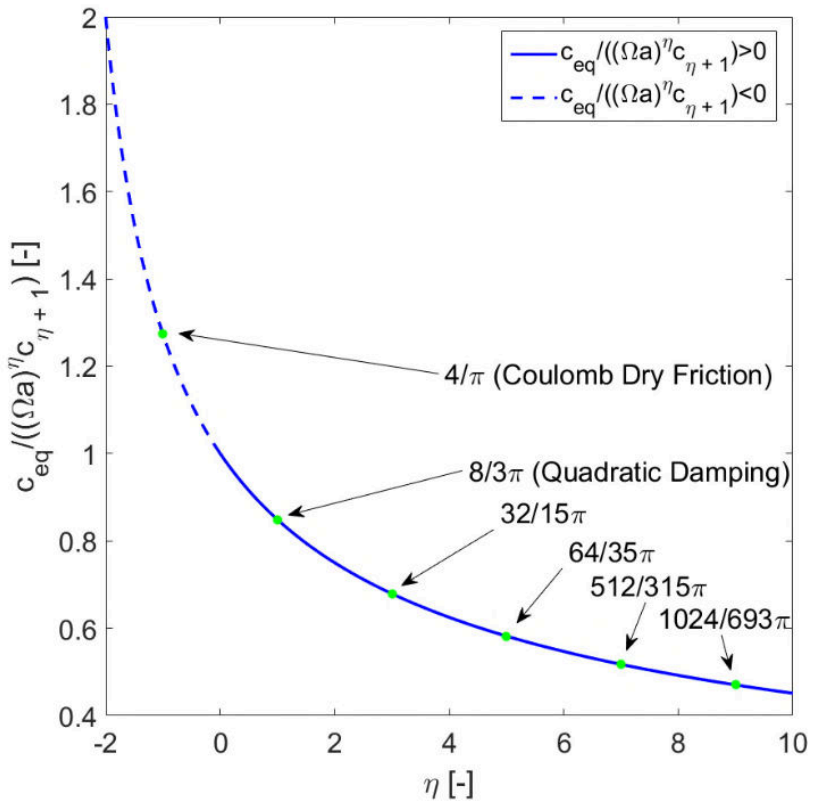

(b)

Figure 4. (a) The equivalent value of the generalised nonlinear stiffness term for increasing $\rho$. (b) The equivalent value of the generalised nonlinear damping term for increasing $\eta$. The blue lines indicate the continuous real values. Green dots indicate even integers for (a) and odd integers for (b). 

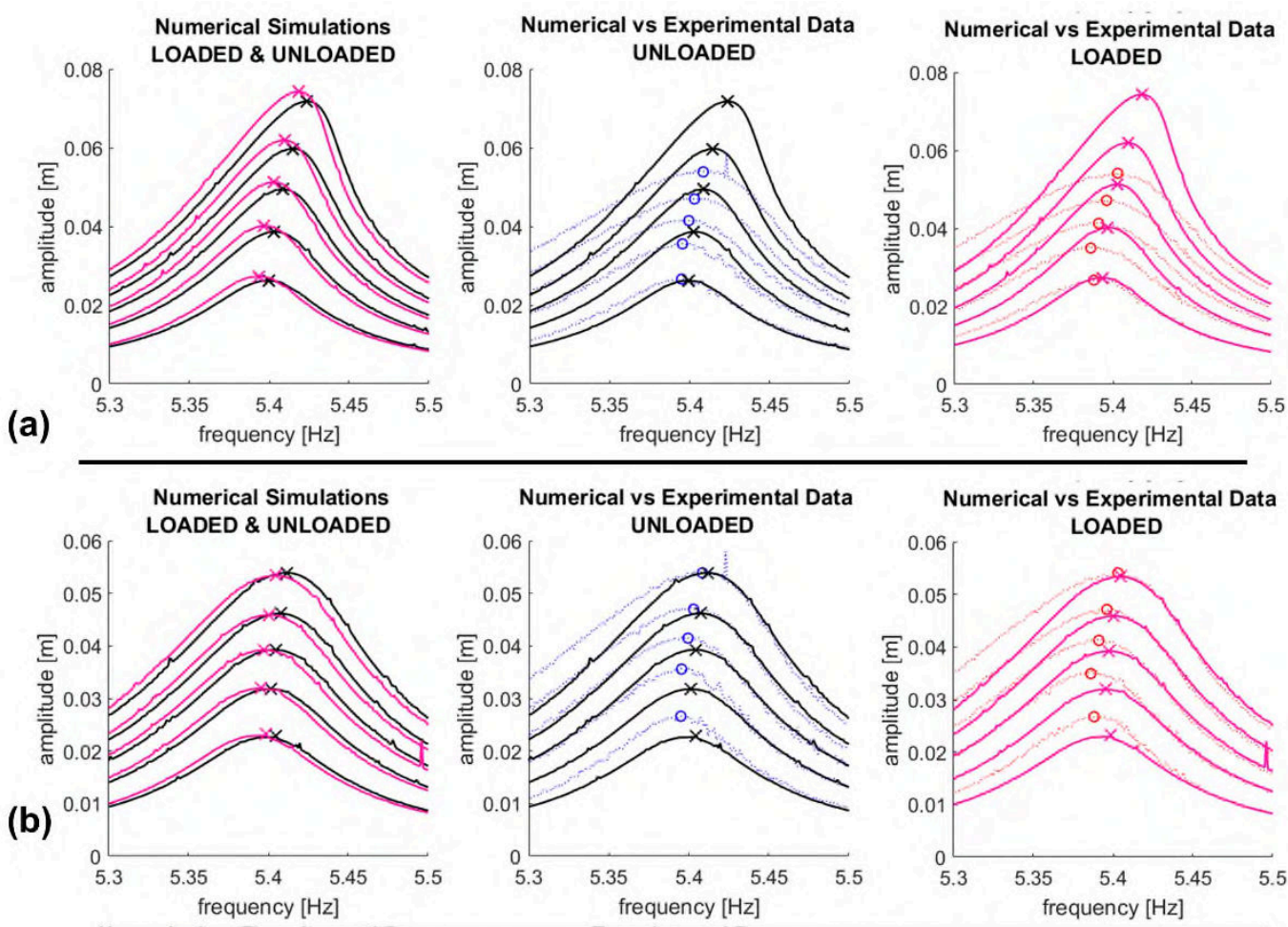

\begin{tabular}{|c|c|}
\hline & $\longrightarrow$ \\
\hline $\begin{array}{l}\text { - Experimental Phase Quadrature - Unloaded } \\
\text { Experimental Data - Loaded }\end{array}$ & $\times$ Mod \\
\hline Experimental Phase Quadrature - Loaded & × Model Phase Quadrature - Loaded \\
\hline
\end{tabular}

Figure 5. Modulation Equation results with Duffing stiffness and quadratic damping: (a) best fit of the model parameters over the lower amplitude levels (b) best fit over the higher amplitude levels. 

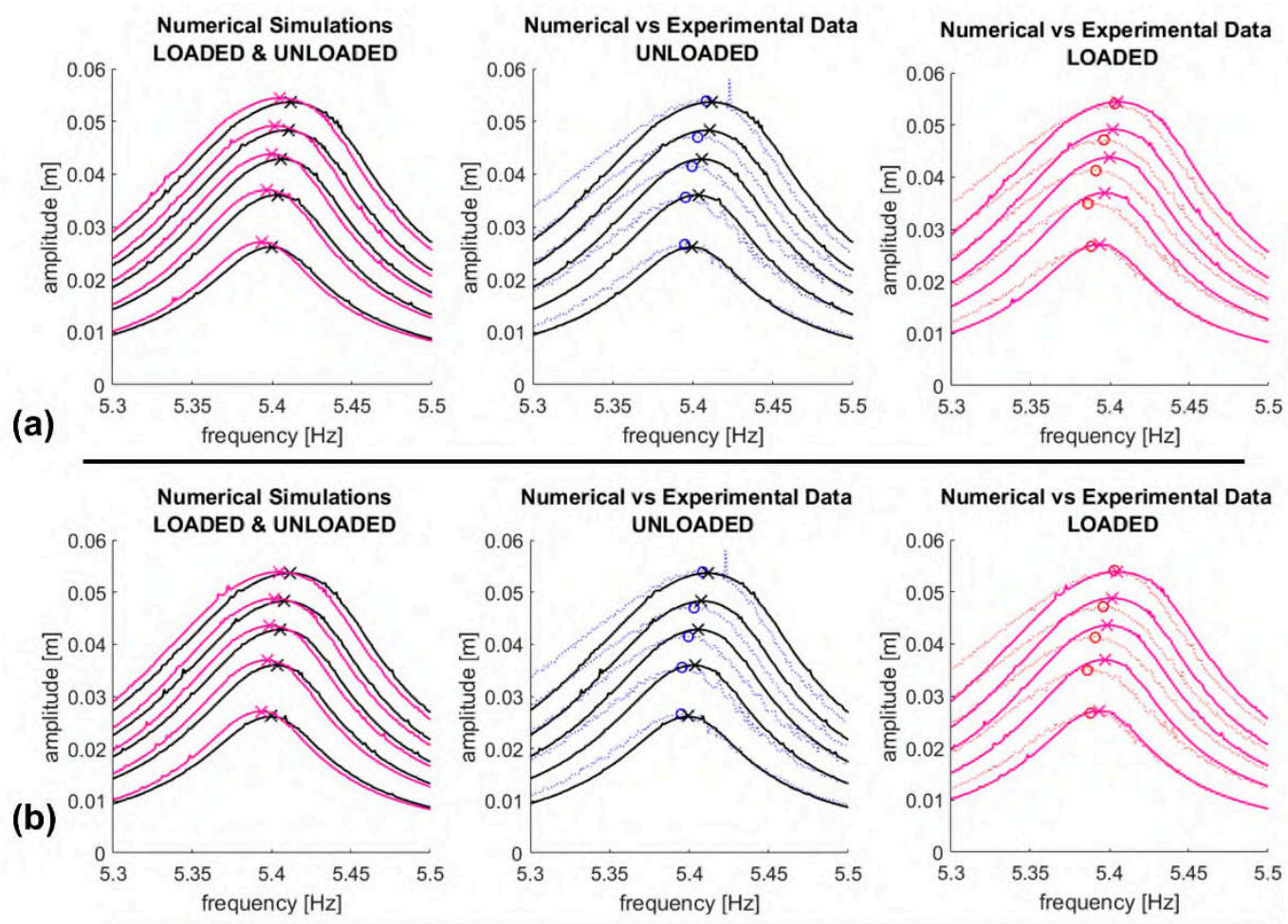

\begin{tabular}{|c|c|c|c|}
\hline \multicolumn{2}{|c|}{ Experimental Data - Unloaded } & \multicolumn{2}{|r|}{ Model Prediction - Unloaded } \\
\hline o & Experimental Phase Quadrature - Unloaded & $\times$ & Model Phase Quadrature - Unloaded \\
\hline & Experimental Data - Loaded & & - Model Prediction - Loaded \\
\hline ० & Experimental Phase Quadrature - Loaded & $x$ & Model Phase Quadrature - Loaded \\
\hline
\end{tabular}

Figure 6. Modulation Equation results: (a) optimised integer values of $\eta$ with Duffing nonlinear stiffness (b) optimised noninteger values of $\eta$ with Duffing nonlinear stiffness. 

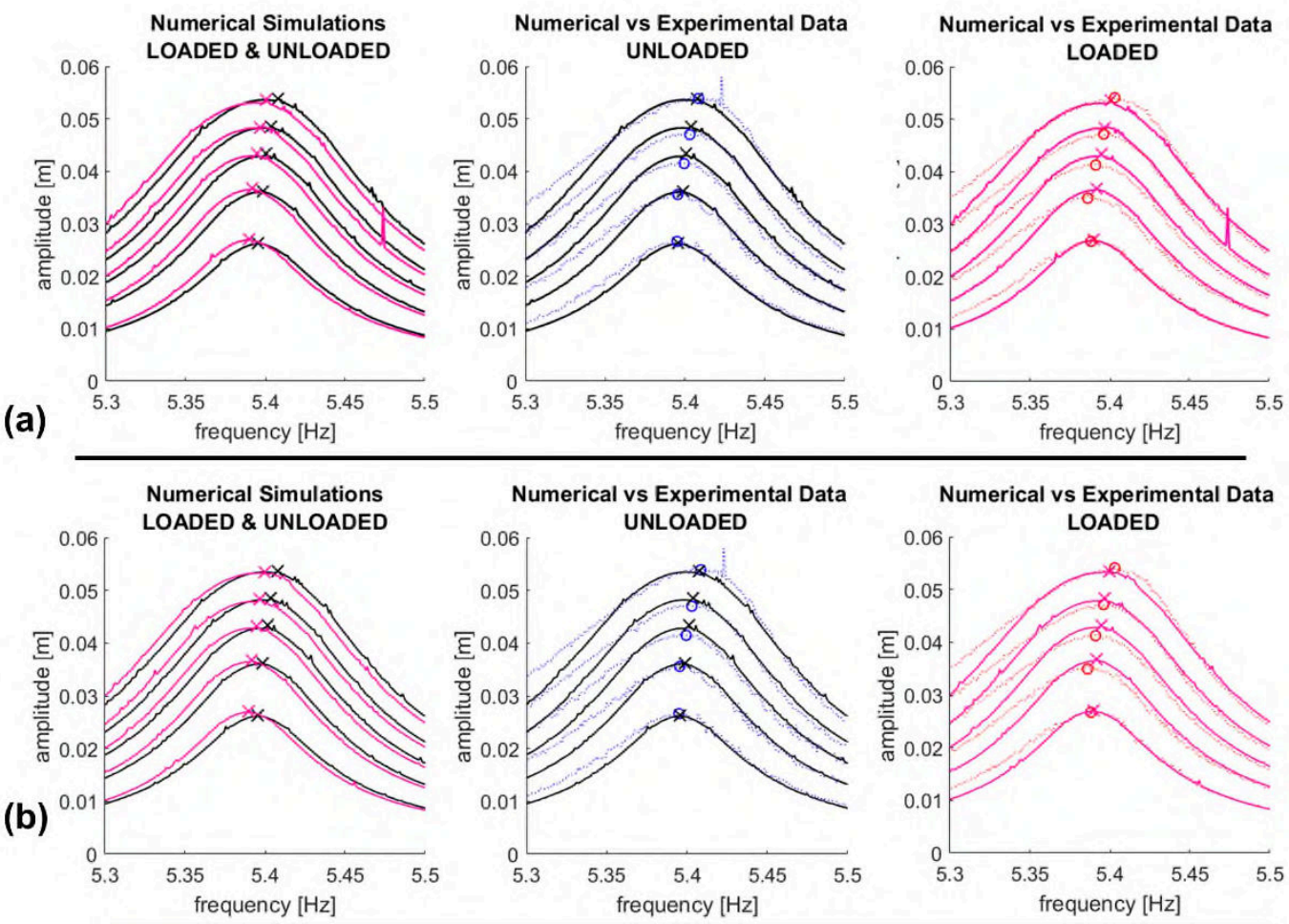

\begin{tabular}{|c|c|c|}
\hline & Experimental Data - Unloaded & - Model Prediction - Unloaded \\
\hline ○ & Experimental Phase Quadrature - Unloaded & Model Phase Quadrature - Unloaded \\
\hline & Experimental Data - Loaded & - Model Prediction - Loaded \\
\hline 0 & Experimental Phase Quadrature - Loaded & Model Phase Quadrature - Loaded \\
\hline
\end{tabular}

Figure 7. Modulation Equation results: (a) optimised integer values of $\eta$ and $\rho(b)$ optimised non-integer values of $\eta$ and $\rho$. 

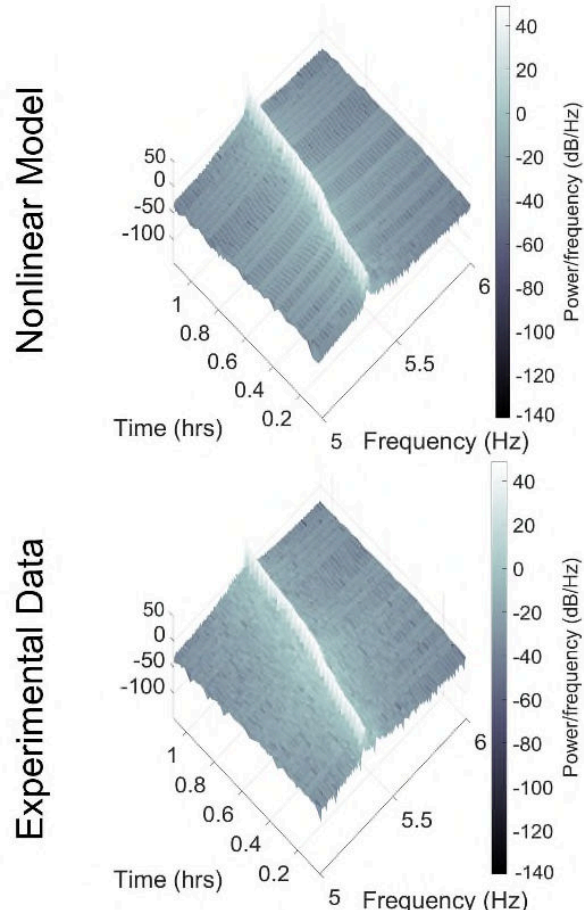

Max Normalised Error: 3.38\%
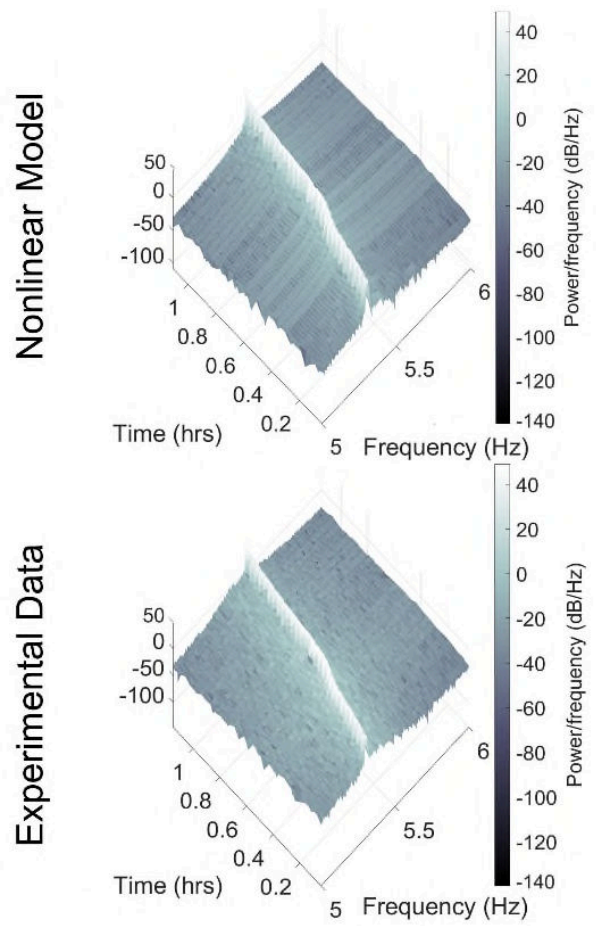

Max Normalised Error: $2.80 \%$
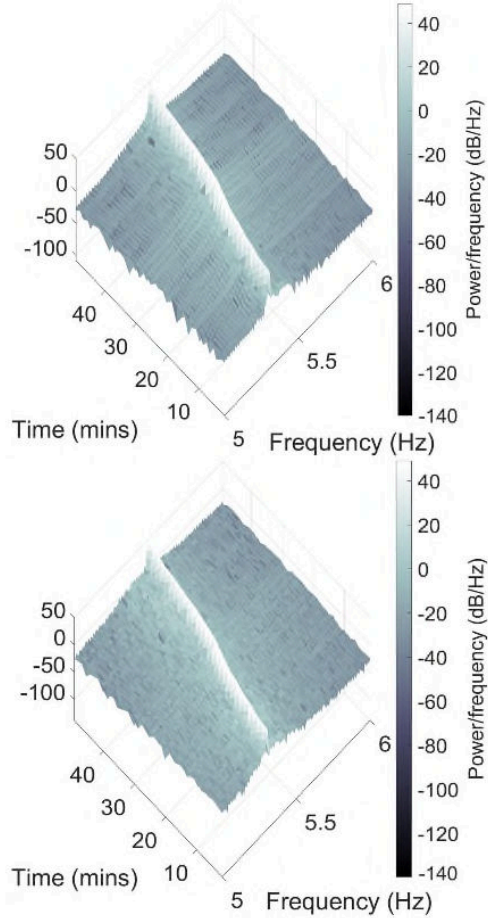

Max Normalised Error: 2.52\%

(a)
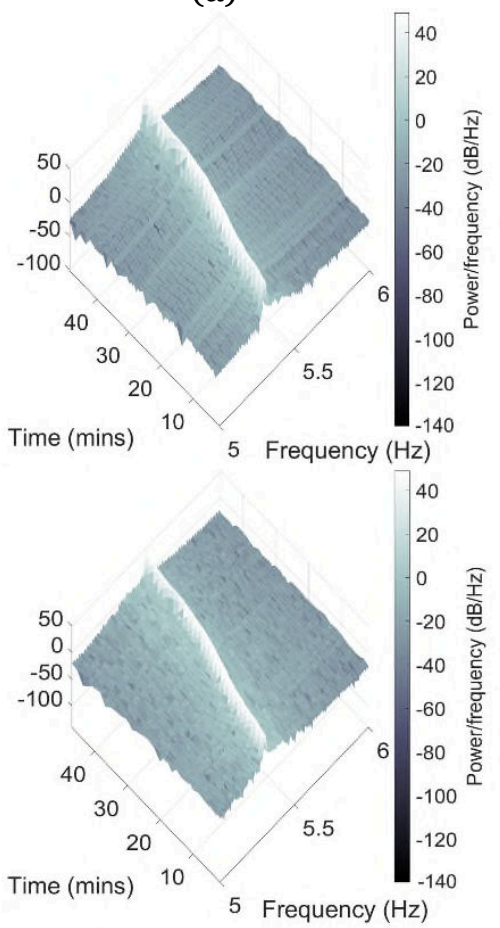

Max Normalised Error: 1.44\%
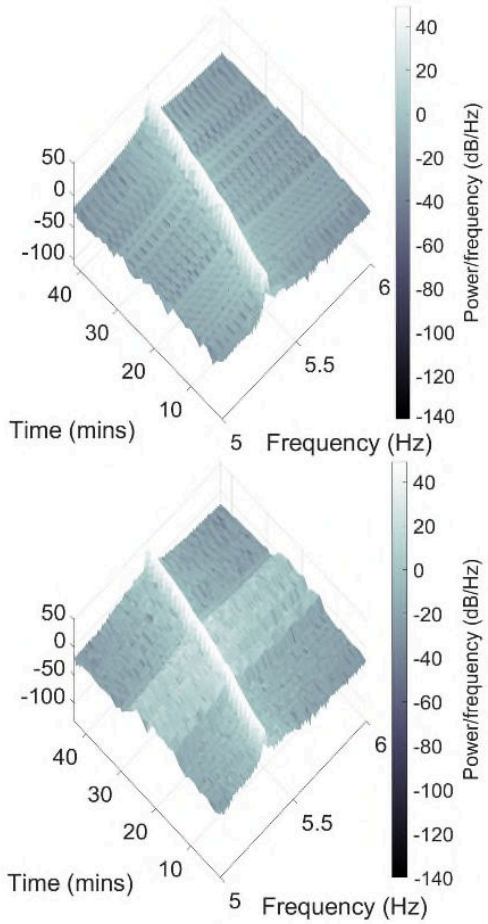

Max Normalised Error: $5.48 \%$
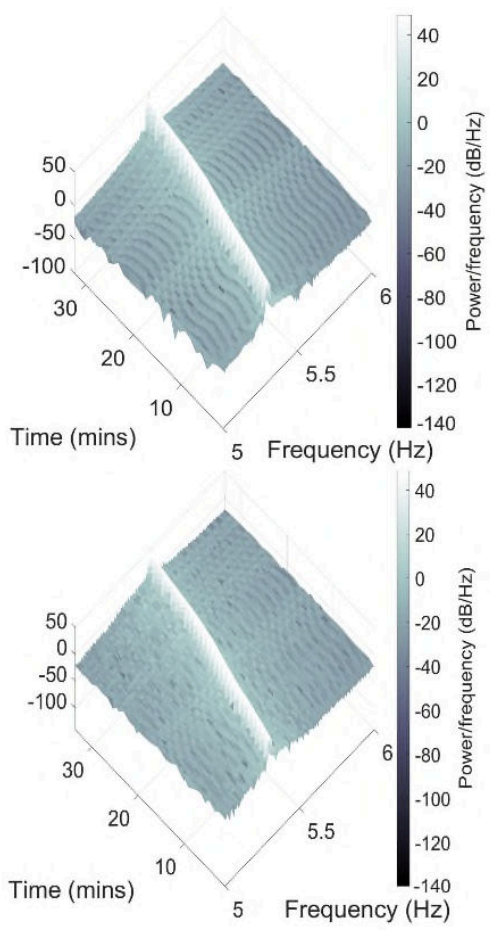

Max Normalised Error: 3.67\%

(b)

Figure 8. 3-D Waterfall Plots of the recorded output and of the time histories obtained from the nonlinear model. (a) Unloaded case, (b) Sensor-loaded case. The recording for the largest input suffered from clipping for the unloaded case. 
Table 1. Mechanical and geometrical properties of the spar.

\begin{tabular}{|c|c|c|}
\hline \multicolumn{2}{|c|}{ Mechanical properties } \\
\hline Density & 2893.07 & $\mathrm{~kg} / \mathrm{m}^{3}$ \\
\hline Young's Modulus & $59.0162 \cdot 10^{3}$ & $\mathrm{MPa}$ \\
\hline Poisson Ratio & 0.2616 & $\mathrm{~mm}$ \\
\hline Free length & Geometrical properties & $\mathrm{mm}$ \\
\hline Thickness & 706 & $\mathrm{~mm}$ \\
\hline $\begin{array}{c}\text { Maximum width at clamped } \\
\text { section }\end{array}$ & 180.00 & $\mathrm{~mm}$ \\
\hline $\begin{array}{c}\text { Mid-length width at the section of } \\
\text { changing tampering (l=258 mm) }\end{array}$ & 56.10 & $\mathrm{~mm}$ \\
\hline Minimum width at the tip section & 17.04 & \\
\hline
\end{tabular}

Table 2. Investigated input amplitudes (both loaded and unloaded configurations).

\begin{tabular}{|c|c|}
\hline Input level & Peak-to-Peak Amplitude \\
\hline & $m / s^{2}$ \\
\hline Level \#1 & 0.82 \\
\hline Level \#2 & 1.24 \\
\hline Level \#3 & 1.62 \\
\hline Level \#4 & 2.00 \\
\hline Level \#5 & 2.46 \\
\hline
\end{tabular}

Table 3. Linear parameters (from Linear System Identification).

\begin{tabular}{|c|c|c|}
\hline Linear parameter & $\begin{array}{l}\text { Without } \\
\text { sensors }\end{array}$ & With sensors \\
\hline$m[k g]$ & 0.26699 & 0.27799 \\
\hline$k[N / m]$ & 306.7878 & 318.6872 \\
\hline$c[N s / m]$ & 0.1164 & 0.1158 \\
\hline
\end{tabular}


Table 4. Summary of the main formulations compared with their classic counterparts.

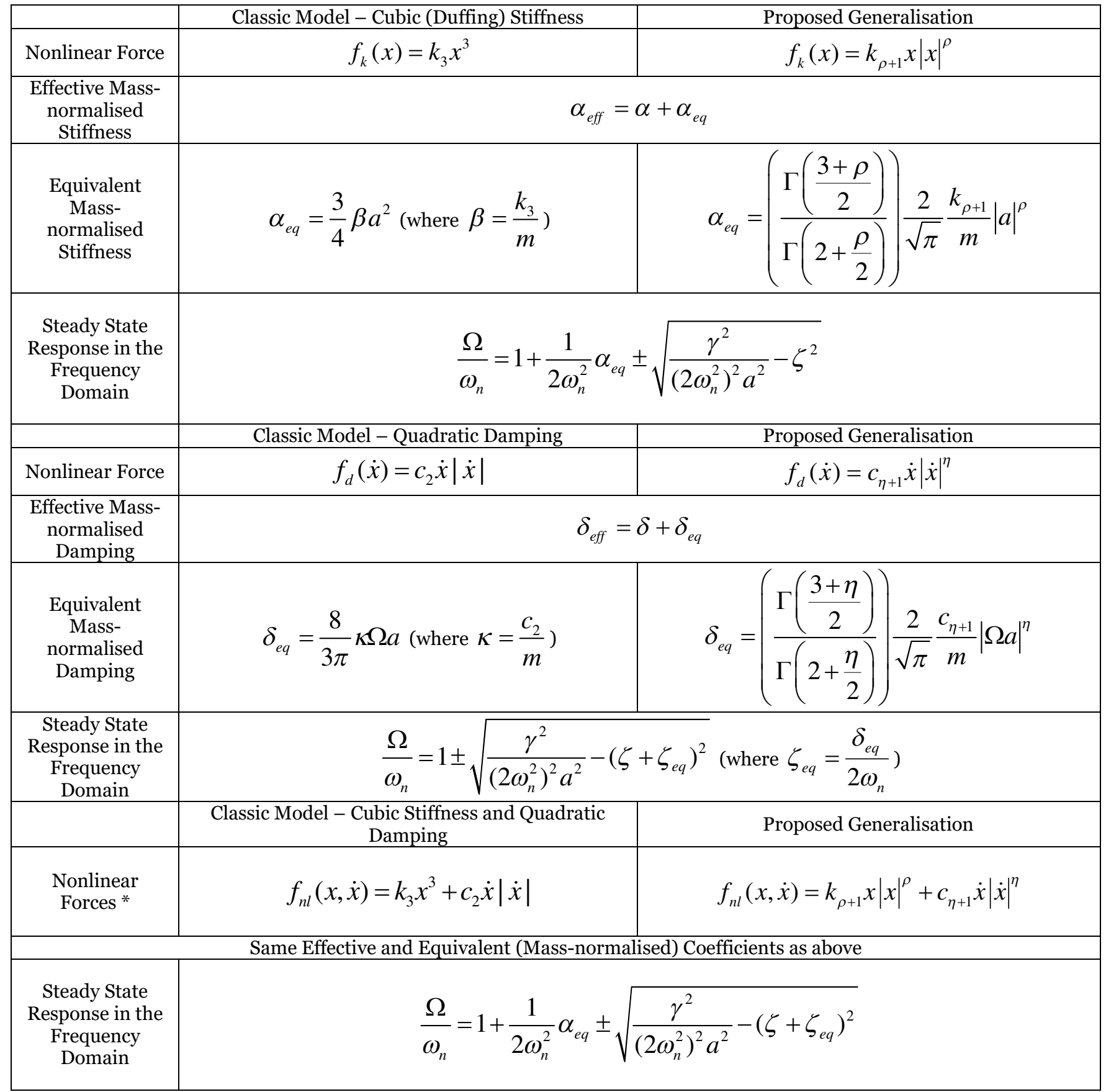

*: the nonlinearities in stiffness and damping are additively separable. 
Table 5. Fitting results - quadratic damping and cubic (Duffing) stiffness.

\begin{tabular}{|c|c|c|c|}
\hline & $\begin{array}{c}\text { Nonlinear } \\
\text { parameter }\end{array}$ & $\begin{array}{c}\text { Without } \\
\text { sensors }\end{array}$ & With sensors \\
\hline \multirow{3}{*}{ Case a } & $k_{3}\left[N / m^{3}\right]$ & 849.99 & 850.00 \\
\cline { 2 - 4 } & $c_{2}\left[N s^{2} / m^{2}\right]$ & 0.0090 & 0.0095 \\
\hline \multirow{3}{*}{ Case b } & $k_{3}\left[N / m^{3}\right]$ & 849.99 & 850.00 \\
\cline { 2 - 5 } & $c_{2}\left[N s^{2} / m^{2}\right]$ & 0.0041 & 0.0048 \\
\hline
\end{tabular}

Table 6. Fitting results - generalised nonlinear damping and Duffing cubic stiffness.

\begin{tabular}{|c|c|c|}
\hline \multirow{2}{*}{$\begin{array}{c}\text { Nonlinear } \\
\text { parameter }\end{array}$} & $\begin{array}{c}\text { Without } \\
\text { sensors }\end{array}$ & With sensors \\
\cline { 2 - 3 } & $\begin{array}{c}\text { Value } \\
\text { (corresponding } \\
\text { integer value) }\end{array}$ & $\begin{array}{c}\text { Value } \\
\text { (corresponding } \\
\text { integer value) }\end{array}$ \\
\hline$c_{\eta+1}\left[\frac{k g \cdot s^{\eta-1}}{m^{\eta}}\right]$ & 0.01580 & 0.01650 \\
\hline$\eta[-]$ & $3.00329(3)$ & $3.11240(3)$ \\
\hline$k_{3}\left[N / m^{3}\right]$ & 849.99 & 850.00 \\
\hline
\end{tabular}

Table 7. Fitting results - generalised nonlinear stiffness and damping.

\begin{tabular}{|c|c|c|c|c|}
\hline & \multicolumn{2}{|c|}{ Without sensors } & \multicolumn{2}{|c|}{ With sensors } \\
\hline Linear parameter & $\begin{array}{c}\text { Value } \\
\text { as pre- } \\
\text { estimated * }\end{array}$ & $\begin{array}{c}\text { Value } \\
\text { as } \\
\text { simultaneously } \\
\text { estimated }\end{array}$ & $\begin{array}{c}\text { Value } \\
\text { as pre- } \\
\text { estimated * }\end{array}$ & $\begin{array}{c}\text { Value } \\
\text { as } \\
\text { simultaneously } \\
\text { estimated }\end{array}$ \\
\hline$m[\mathrm{~kg}]$ & 0.26699 & 0.26630 & 0.27799 & 0.27802 \\
\hline$k[N / m]$ & 306.7878 & 305.6820 & 318.6872 & 318.4451 \\
\hline$c[N s / m]$ & 0.1164 & 0.1132 & 0.1158 & 0.1156 \\
\hline $\begin{array}{l}\text { Nonlinear } \\
\text { parameter }\end{array}$ & \multicolumn{4}{|c|}{ Non-integer value (corresponding integer indicated in brackets) } \\
\hline$c_{\eta+1}\left[\frac{k g \cdot s^{\eta-1}}{m^{\eta}}\right]$ & 0.01591 & 0.01590 & 0.01918 & 0.01917 \\
\hline$\eta[-]$ & $3.03099(3)$ & $3.02180(3)$ & $3.05177(3)$ & $3.00569(3)$ \\
\hline$k_{\rho+1}\left[\frac{k g}{m^{\rho} s^{2}}\right]$ & 85000 & 85000 & 84999 & 85000 \\
\hline$\rho[-]$ & $4.00220(4)$ & 4.04629 & $4.00087(4)$ & $4.04803(4)$ \\
\hline
\end{tabular}

*: as also already reported in Table 3 . 
Table 8. Comparison of simulated output and experimental data - generalised nonlinear stiffness and damping.

\begin{tabular}{|c|c|c|c|c|c|c|c|c|c|c|}
\hline \multicolumn{11}{|c|}{ Unloaded configuration } \\
\hline & \multicolumn{2}{|c|}{ Experimental } & \multicolumn{4}{|c|}{ Non-integer values of $\eta, \rho$} & \multicolumn{4}{|c|}{ Integer values of $\eta, \rho$} \\
\hline $\begin{array}{l}\text { Input P-P } \\
\text { amplitude } \\
\qquad \gamma\end{array}$ & $\begin{array}{c}\text { Amplitude } \\
\text { at phase } \\
\text { quadrature } \\
\bar{a}_{P Q}\end{array}$ & $\begin{array}{c}\text { Frequency } \\
\text { at phase } \\
\text { quadrature } \\
\bar{f}_{P Q}(\gamma)\end{array}$ & $a_{P Q}$ & $\Delta a_{P Q}$ & $f_{P Q}(\gamma)$ & $\Delta f_{P Q}(\gamma)$ & $a_{P Q}$ & $\Delta a_{P Q}$ & $f_{P Q}(\gamma)$ & $\Delta f_{P Q}(\gamma)$ \\
\hline$\left[\mathrm{m} / \mathrm{s}^{2}\right]$ & {$[m]$} & {$[\mathrm{Hz}]$} & {$[m]$} & {$[\%]$} & {$[\mathrm{Hz}]$} & {$[\%]$} & {$[m]$} & {$[\%]$} & {$[\mathrm{Hz}]$} & {$[\%]$} \\
\hline 0.82 & 0.02662 & 5.3950 & 0.02630 & +1.20 & 5.3955 & -0.01 & 0.02633 & +1.09 & 5.3955 & -0.01 \\
\hline 1.24 & 0.03558 & 5.3955 & 0.03620 & -1.74 & 5.3985 & -0.06 & 0.03624 & -1.85 & 5.3985 & -0.06 \\
\hline 1.62 & 0.04147 & 5.3995 & 0.04341 & -4.68 & 5.4005 & -0.02 & 0.04341 & -4.68 & 5.4005 & -0.02 \\
\hline 2.00 & 0.04699 & 5.4030 & 0.04845 & -3.11 & 5.4035 & -0.01 & 0.04852 & -3.26 & 5.4035 & -0.01 \\
\hline 2.46 & 0.05387 & 5.4085 & 0.05363 & +0.45 & 5.4075 & +0.02 & 0.05375 & +0.22 & 5.4075 & +0.02 \\
\hline \multicolumn{11}{|c|}{ Loaded configuration } \\
\hline & \multicolumn{2}{|c|}{ Experimental } & \multicolumn{4}{|c|}{ Non-integer values of $\eta, \rho$} & \multicolumn{4}{|c|}{ Integer values of $\eta, \rho$} \\
\hline $\begin{array}{l}\text { Input P-P } \\
\text { amplitude } \\
\qquad \gamma\end{array}$ & $\begin{array}{l}\text { Amplitude } \\
\text { at phase } \\
\text { quadrature } \\
\bar{a}_{P Q}\end{array}$ & $\begin{array}{c}\text { Frequency } \\
\text { at phase } \\
\text { quadrature } \\
\bar{f}_{P Q}(\gamma)\end{array}$ & $a_{P Q}$ & $\Delta a_{P Q}$ & $f_{P Q}(\gamma)$ & $\Delta f_{P Q}(\gamma)$ & $a_{P Q}$ & $\Delta a_{P Q}$ & $f_{P Q}(\gamma)$ & $\Delta f_{P Q}(\gamma)$ \\
\hline$\left[\mathrm{m} / \mathrm{s}^{2}\right]$ & {$[m]$} & {$[\mathrm{Hz}]$} & {$[m]$} & {$[\%]$} & {$[\mathrm{Hz}]$} & {$[\%]$} & {$[m]$} & {$[\%]$} & {$[\mathrm{Hz}]$} & {$[\%]$} \\
\hline 0.82 & 0.02672 & 5.3880 & 0.02713 & -1.53 & 5.3895 & -0.03 & 0.02713 & -1.53 & 5.3895 & -0.03 \\
\hline 1.24 & 0.03493 & 5.3860 & 0.03680 & -5.35 & 5.3915 & -0.10 & 0.03681 & -5.38 & 5.3915 & -0.10 \\
\hline 1.62 & 0.04125 & 5.3910 & 0.04323 & -4.80 & 5.3945 & -0.06 & 0.04343 & -5.28 & 5.3945 & -0.06 \\
\hline 2.00 & 0.04715 & 5.3960 & 0.04836 & -2.57 & 5.3965 & -0.01 & 0.04841 & -2.67 & 5.3965 & -0.01 \\
\hline 2.46 & 0.05410 & 5.4030 & 0.05352 & -1.07 & 5.3995 & +0.06 & 0.05364 & +0.85 & 5.4005 & +0.05 \\
\hline
\end{tabular}


2020-12-26

\section{A generalised power-law formulation for} the modelling of damping and stiffness nonlineariti

Civera, Marco

Elsevier

Civera M, Grivet-Talocia S, Surace C, Zanotti Fragonara L. (2021) A generalised power-law formulation for the modelling of damping and stiffness nonlinearities. Mechanical Systems and Signal Processing, Volume 153, May 2021, Article number 107531

https://doi.org/10.1016/j.ymssp.2020.107531

Downloaded from Cranfield Library Services E-Repository 